\title{
The blow-up of critical anisotropic equations with critical directions
}

\author{
Jérôme Vétois
}

\begin{abstract}
We investigate blow-up theory for doubly critical anisotropic problems in bounded domains of the Euclidean space.

Mathematics Subject Classification (2000). 35J60 Nonlinear PDE of elliptic type.
\end{abstract}

\section{Introduction}

Anisotropic operators modelize directionally dependent phenomena. In this paper, we consider problems posed on domains in the Euclidean space $\mathbb{R}^{n}$ in dimension $n \geq 2$, and we provide ourselves with an anisotropic configuration $\vec{p}=\left(p_{1}, \ldots, p_{n}\right)$ with $p_{i}>1$ for all $i=1, \ldots, n$. We define the anisotropic Laplace operator $\Delta_{\vec{p}}$ by

$$
\Delta_{\vec{p}} u=\sum_{i=1}^{n} \frac{\partial}{\partial x_{i}} \nabla_{x_{i}}^{p_{i}} u
$$

where $\nabla_{x_{i}}^{p_{i}} u=\left|\partial u / \partial x_{i}\right|^{p_{i}-2} \partial u / \partial x_{i}$ for all $i=1, \ldots, n$. Nonlinear equations of the type $\Delta_{\vec{p}} u=f(\cdot, u)$ appear in several places in the literature. They appear, for instance, in biology, see Bendahmane-Karlsen [11] and BendahmaneLanglais-Saad [13], as a model describing the spread of an epidemic disease in heterogeneous environments. They also emerge, see Antontsev-Díaz-Shmarev [5] and Bear [10], from the mathematical description of the dynamics of fluids with different conductivities in different directions.

We consider anisotropic problems of critical growth of the type

$$
\left\{\begin{array}{l}
-\Delta_{\vec{p}} u=\lambda|u|^{p^{*}-2} u+f(\cdot, u) \text { in } \Omega, \\
u \in D^{1, \vec{p}}(\Omega),
\end{array}\right.
$$

where $\Delta_{\vec{p}}$ is as in (1.1), $\Omega$ is a domain of $\mathbb{R}^{n}, D^{1, \vec{p}}(\Omega)$ is the anisotropic Sobolev space defined as the completion of the vector space of all smooth 
functions with compact support in $\Omega$ with respect to the norm $\|u\|_{D^{1, \vec{p}}(\Omega)}=$ $\sum_{i=1}^{n}\left\|\partial u / \partial x_{i}\right\|_{L^{p_{i}(\Omega)}}, p^{*}$ is the critical Sobolev exponent [see (1.4) below], $\lambda$ is a positive real number, and $f$ is a Caratheodory function in $\Omega \times \mathbb{R}$ satisfying the growth condition

$$
|f(\cdot, u)| \leq C\left(|u|^{q-1}+1\right) \quad \text { a.e. in } \Omega
$$

for some real number $q$ in $\left(1, p^{*}\right)$ and for some positive constant $C$ independent of $u$.

We are concerned with the doubly critical situation $p_{+}=p^{*}$, where $p_{+}=$ $\max \left(p_{1}, \ldots, p_{n}\right)$ is the maximum value of the anisotropic configuration and $p^{*}$ is as above the critical Sobolev exponent for the embeddings of the anisotropic Sobolev space $D^{1, \vec{p}}(\Omega)$ into Lebesgue spaces. In this setting, not only the nonlinearity has critical growth, but the operator itself has critical growth in particular directions of the Euclidean space. As a remark, the notion of critical direction is a pure anisotropic notion which does not exist when dealing with the Laplace operator or the $p$-Laplace operator. Given $i=1, \ldots, n$, the $i$ th direction is said to be critical if $p_{i}=p^{*}$, resp. subcritical if $p_{i}<p^{*}$. Critical directions induce a failure in the rescaling invariance rule associated with (1.2).

Given an anisotropic configuration $\vec{p}$ satisfying $\sum_{i=1}^{n} 1 / p_{i}>1$ and $p_{j} \leq$ $n /\left(\sum_{i=1}^{n} \frac{1}{p_{i}}-1\right)$ for all $j=1, \ldots, n$, the critical Sobolev exponent is equal to

$$
p^{*}=\frac{n}{\sum_{i=1}^{n} \frac{1}{p_{i}}-1} .
$$

Possible references on anisotropic Sobolev spaces are Besov [14], HaškovecSchmeiser [38], Kruzhkov-Kolodü[40], Kruzhkov-Korolev [41], Lu [47], Nikol'skiị [53], Rákosník [54,55], and Troisi [65].

We aim in describing the asymptotic behaviors in energy space of Palais-Smale sequences associated with problem (1.2). Before stating our main result, let us fix some notations. For any $\mu>0$ and any point $a=\left(a_{1}, \ldots, a_{n}\right)$ in $\mathbb{R}^{n}$, we define the affine transformation $\tau_{\mu, a}^{\vec{p}}: \mathbb{R}^{n} \rightarrow \mathbb{R}^{n}$ by

$$
\tau_{\mu, a}^{\vec{p}}\left(x_{1}, \ldots, x_{n}\right)=\left(\mu^{\frac{p_{1}-p^{*}}{p_{1}}}\left(x_{1}-a_{1}\right), \ldots, \mu^{\frac{p_{n}-p^{*}}{p_{n}}}\left(x_{n}-a_{n}\right)\right) .
$$

As is easily checked, (1.5) provides a general rescaling invariance rule associated with problem (1.2) with $f \equiv 0$. In particular, $u$ solves $-\Delta_{\vec{p}} u=|u|^{p^{*}-2} u$ in $\Omega$ if and only if the function $v=\mu^{-1} u \circ\left(\tau_{\mu, a}^{\vec{p}}\right)^{-1}$ solves $-\Delta_{\vec{p}} v=|v|^{p^{*}-2} v$ in $\tau_{\mu, a}^{\vec{p}}(\Omega)$, where

$$
\left(\tau_{\mu, a}^{\vec{p}}\right)^{-1}\left(x_{1}, \ldots, x_{n}\right)=\left(\mu^{\frac{p^{*}-p_{1}}{p_{1}}} x_{1}+a_{1}, \ldots, \mu^{\frac{p^{*}-p_{n}}{p_{n}}} x_{n}+a_{n}\right) .
$$

Given $\left(\mu_{\alpha}\right)_{\alpha}$ a sequence of positive real numbers converging to $0,\left(x_{\alpha}\right)_{\alpha}$ a converging sequence in $\mathbb{R}^{n}, \lambda$ a positive real number, $U$ a nonempty, open subset of $\mathbb{R}^{n}$, and $u$ a nontrivial solution in $D^{1, \vec{p}}(U)$ of the problem

$$
\left\{\begin{array}{l}
-\Delta_{\vec{p}} u=\lambda|u|^{p^{*}-2} u \text { in } U, \\
u \in D^{1, \vec{p}}(U),
\end{array}\right.
$$


where $\Delta_{\vec{p}}$ is as in (1.1), we call $\vec{p}$-bubble of centers $\left(x_{\alpha}\right)_{\alpha}$, weights $\left(\mu_{\alpha}\right)_{\alpha}$, multiplier $\lambda$, domain $U$, and profile $u$, the sequence $\left(B_{\alpha}\right)_{\alpha}$ defined by

$$
B_{\alpha}=\frac{1}{\mu_{\alpha}} u \circ \tau_{\mu_{\alpha}, x_{\alpha}}^{\vec{p}}
$$

for all $\alpha$, where $\tau_{\mu_{\alpha}, x_{\alpha}}^{\vec{p}}$ is as in (1.5). One can find existence and regularity results for problem (1.6) in Vétois [68]. In the following, we implicitly extend profiles of bubbles by 0 outside of their domains so as to regard them as functions in $D^{1, \vec{p}}\left(\mathbb{R}^{n}\right)$. With the above notations, we define the energy $E\left(B_{\alpha}\right)$ of a $\vec{p}$-bubble $\left(B_{\alpha}\right)_{\alpha}$ by

$$
E\left(B_{\alpha}\right)=\sum_{i=1}^{n} \frac{1}{p_{i}} \int_{\mathbb{R}^{n}}\left|\frac{\partial u}{\partial x_{i}}\right|^{p_{i}} d x-\frac{\lambda}{p^{*}} \int_{\mathbb{R}^{n}}|u|^{p^{*}} d x=\sum_{i=1}^{n} \frac{p^{*}-p_{i}}{p^{*} p_{i}} \int_{\mathbb{R}^{n}}\left|\frac{\partial u}{\partial x_{i}}\right|^{p_{i}} d x
$$

the second equality in (1.7) being obtained by testing (1.6) with $u$ and integrating by parts.

We approximate problem (1.2) with the problems

$$
\left\{\begin{array}{l}
-\Delta_{\vec{p}} u=\lambda_{\alpha}|u|^{r_{\alpha}-2} u+f(\cdot, u) \quad \text { in } \Omega, \\
u \in D^{1, \vec{p}}(\Omega)
\end{array}\right.
$$

where $\Delta_{\vec{p}}$ is as in (1.1), $\left(\lambda_{\alpha}\right)_{\alpha}$ is a sequence of positive real numbers converging to $\lambda$, and $\left(r_{\alpha}\right)_{\alpha}$ is a sequence of real numbers in $\left(1, p^{*}\right]$ converging to $p^{*}$. Associated with problems (1.2) and (1.8), we define the functionals $I_{\alpha}$ and $I_{\infty}$ in $D^{1, \vec{p}}(\Omega)$ by

$$
I_{\alpha}(u)=\sum_{i=1}^{n} \frac{1}{p_{i}} \int_{\Omega}\left|\frac{\partial u}{\partial x_{i}}\right|^{p_{i}} d x-\int_{\Omega} F(x, u) d x-\frac{\lambda_{\alpha}}{r_{\alpha}} \int_{\Omega}|u|^{r_{\alpha}} d x
$$

and

$$
I_{\infty}(u)=\sum_{i=1}^{n} \frac{1}{p_{i}} \int_{\Omega}\left|\frac{\partial u}{\partial x_{i}}\right|^{p_{i}} d x-\int_{\Omega} F(x, u) d x-\frac{\lambda}{p^{*}} \int_{\Omega}|u|^{p^{*}} d x,
$$

where $F(x, u)=\int_{0}^{u} f(x, s) d s$. We say that a sequence $\left(u_{\alpha}\right)_{\alpha}$ in $D^{1, \vec{p}}(\Omega)$ is Palais-Smale for the functionals $\left(I_{\alpha}\right)_{\alpha}$ if there hold $\left|I_{\alpha}\left(u_{\alpha}\right)\right| \leq C$ for some positive constant $C$ independent of $\alpha$ and $D I_{\alpha}\left(u_{\alpha}\right) \rightarrow 0$ in $D^{1, \vec{p}}(\Omega)^{\prime}$ as $\alpha \rightarrow+\infty$. We say that $\left(u_{\alpha}\right)_{\alpha}$ is a bounded Palais-Smale sequence for the functionals $\left(I_{\alpha}\right)_{\alpha}$ if $\left(u_{\alpha}\right)_{\alpha}$ is moreover bounded in $D^{1, \vec{p}}(\Omega)$.

Theorem 1.1 below is referred to as a bubble tree decomposition. With this result, we provide a complete description of the asymptotic behaviors in $D^{1, \vec{p}}(\Omega)$ of bounded Palais-Smale sequences for the functionals $\left(I_{\alpha}\right)_{\alpha}$ defined in (1.9). Bubble tree decompositions were obtained by Struwe [62] for the equation $-\Delta u=u^{2^{*}-1}$, where $\Delta$ is the classical Laplace operator and $2^{*}=$ $2 n /(n-2)$ is the critical Sobolev exponent. Related references to Struwe [62] are Brézis-Coron [17], Lions [45,46], Sacks-Uhlenbeck [58], Schoen [60], and Wente [70]. We also refer to El Hamidi-Vétois [29] for an extension of Struwe's result to the anisotropic Laplace operator in case $p_{+}<p^{*}$. Here, we 
treat the more involved case of critical directions $p_{+}=p^{*}$. The definition of asymptotically $\vec{p}$-stable domains is postponed to Sect. 3. Roughly speaking, asymptotically stable domains are domains which, in the limit of the rescalings, still satisfy the segment property. This notion, see El Hamidi-Vétois [29] and Vétois [67], turns out to be fundamentally associated with the question of proving bubble tree decompositions for anisotropic critical equations. We state our result as follows.

Theorem 1.1. Let $n \geq 3$ and $\vec{p}=\left(p_{1}, \ldots, p_{n}\right)$, and assume that $\sum_{i=1}^{n} 1 / p_{i}>1$ and $p_{+}=p^{*}$. Let $\Omega$ be an asymptotically $\vec{p}$-stable, bounded domain of $\mathbb{R}^{n}$, and $f$ be a Caratheodory function in $\Omega \times \mathbb{R}$ satisfying the growth condition (1.3). Let $\left(\lambda_{\alpha}\right)_{\alpha}$ be a sequence of positive real numbers converging to $\lambda$, and $\left(r_{\alpha}\right)_{\alpha}$ be a sequence of real numbers in $\left(1, p_{+}\right]$converging to $p_{+}$. For any bounded Palais-Smale sequence $\left(u_{\alpha}\right)_{\alpha}$ for the functionals $\left(I_{\alpha}\right)_{\alpha}$ defined in (1.9), there exist a solution $u_{\infty}$ of problem (1.2), a natural number $k$, and for any $j=$ $1, \ldots, k, a \vec{p}$-bubble $\left(B_{\alpha}^{j}\right)_{\alpha}$ of weights $\left(\mu_{\alpha}^{j}\right)_{\alpha}$ and multiplier $\widetilde{\lambda}_{j}$ satisfying $\left(\mu_{\alpha}^{j}\right)^{p_{+}-r_{\alpha}} \rightarrow \widetilde{\lambda}_{j} / \lambda$ as $\alpha \rightarrow+\infty$, such that, up to a subsequence, there holds

$$
u_{\alpha}=u_{\infty}+\sum_{j=1}^{k} B_{\alpha}^{j}+R_{\alpha}
$$

for all $\alpha$, where $R_{\alpha} \rightarrow 0$ in $D^{1, \vec{p}}\left(\mathbb{R}^{n}\right)$ as $\alpha \rightarrow+\infty$. Moreover, there holds

$$
I_{\alpha}\left(u_{\alpha}\right)=I_{\infty}\left(u_{\infty}\right)+\sum_{j=1}^{k} E\left(B_{\alpha}^{j}\right)+\mathrm{o}(1)
$$

as $\alpha \rightarrow+\infty$, where $E, I_{\alpha}$, and $I_{\infty}$ are as in (1.7), (1.9), and (1.10). If in addition the functions $u_{\alpha}$ are nonnegative, then $u_{\infty}$ and $\left(B_{\alpha}^{1}\right)_{\alpha}, \ldots,\left(B_{\alpha}^{k}\right)_{\alpha}$ are also nonnegative.

We give examples in Sect. 2, see Corollary 2.5, of Palais-Smale sequences which blow up, namely which develop at least one $\vec{p}$-bubble in their decompositions.

The analogue of Theorem 1.1 for the case $p_{+}<p^{*}$ was established in El Hamidi-Vétois [29]. The essential difference in our doubly critical setting $p_{+}=p^{*}$ lies in the effect of the transformation (1.5). Indeed, contrary to the case $p_{+}<p^{*}$ where all directions are subcritical, the domain is only rescaled with respect to some directions, the noncritical ones, in case $p_{+}=p^{*}$. Therefore, we observe a concentration phenomenon on affine subspaces of $\mathbb{R}^{n}$ spanned by critical directions. For instance, Fig. 1 below illustrates the rescaling of a three-dimensional ball, the first two directions being noncritical and the third direction being critical. Together with the rescaling of the domain, the equation in (1.2) is rescaled. At the limit, we find the purely invariant problem (1.6). This problem is studied in Vétois [68]. We established in [68] an existence result on cylindric domains and a general regularity result. We refer to Sect. 2, see Corollary 2.2, for examples of solutions of the limit problem (1.6). 

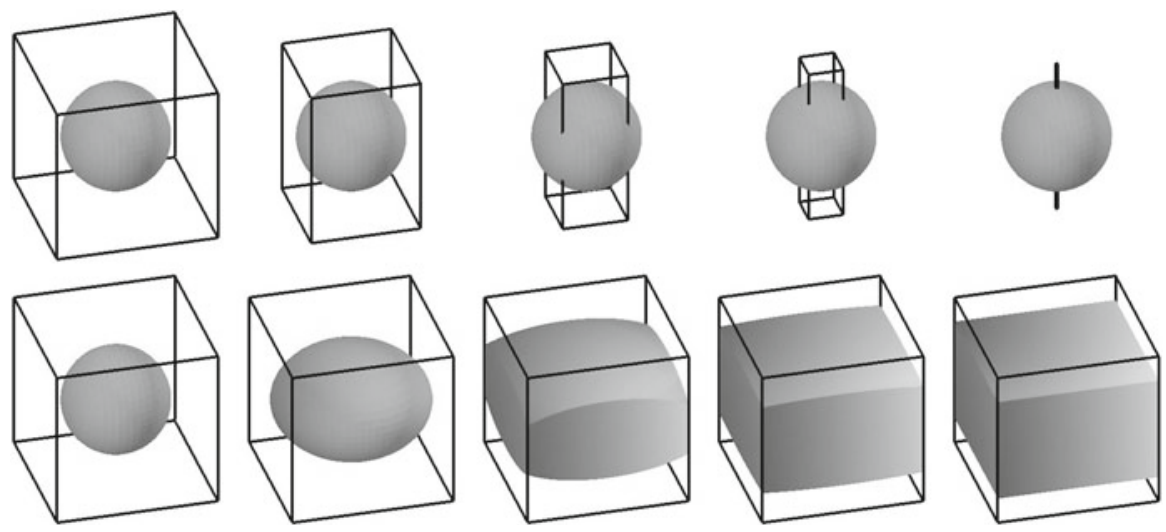

Figure 1. Rescaling of a ball $\left(n=3, p_{1}=p_{2}=1.5, p_{3}=6\right)$. The first line describes the scale in the rescaling. The second line describes the deformation of the domain

As a last remark concerning the case $p_{+}=p^{*}$, it is a borderline case for the boundedness of Palais-Smale sequences. Indeed, while any Palais-Smale sequence is bounded in case $p_{+}<p^{*}$ for nonlinearities of the type $|u|^{r_{\alpha}-2} u+$ $f(x, u), r_{\alpha} \rightarrow p^{*}$ (see El Hamidi-Vétois [29]), we get examples of unbounded Palais-Smale sequences in case $p_{+}>p^{*}$ for the nonlinearity $|u|^{p_{+}-2} u$ (see Fragalà-Gazzola-Kawohl [31]).

Theorem 1.1 should be seen as a general key step in the analysis of blowup for critical anisotropic equations. We have in view the numerous applications of bubble tree decompositions which have been developed for the equation $-\Delta u=u^{2^{*}-1}$. Among other applications, bubble tree decompositions turn out to be key points in the use of topological arguments such as LusternikSchnirelmann equivariant categories. They also turn out to be key points in the analysis of ruling out bubbling and proving compactness of solutions. Possible references in book form on these subjects are Druet-Hebey-Robert [26], Ghoussoub [36], and Struwe [63].

In the isotropic configuration where $p_{i}=p$ for all $i=1, \ldots, n$, there holds $p<p^{*}$ and all directions are subcritical. In this particular situation, the operator (1.2) is comparable, though slightly different, to the $p$-Laplace operator $\Delta_{p}=\operatorname{div}\left(|\nabla u|^{p-2} \nabla u\right)$. Possible references on critical $p$-Laplace equations are Alves [2], Alves-Ding [3], Arioli-Gazzola [8], Demengel-Hebey [22,23], Filippucci-Pucci-Robert [30], Gazzola [35], Guedda-Veron [37], Saintier [59], and Yan [71]. Needless to say, the above list does not pretend to exhaustivity.

In case $p_{+}<p^{*}$, namely when all directions are subcritical, anisotropic equations with critical nonlinearities have been investigated by Alves-El Hamidi [4], El Hamidi-Rakotoson [27,28], El Hamidi-Vétois [29], FragalàGazzola-Kawohl [31], Fragalà-Gazzola-Lieberman [32], and Vétois [67]. Other possible references on anisotropic problems like (1.1) are Antontsev-Shmarev $[6,7]$, Bendahmane-Karlsen [11,12], Bendahmane-Langlais-Saad [13], 
Boccardo-Gallouët-Marcellini [15], Boccardo-Marcellini-Sbordone [16], Cianchi [20], D’Ambrosio [21], Di Castro [24], Di Castro-Montefusco [25], GarcíaMelián-Rossi-Sabina de Lis [34], Kolodı̋[39], Li [42], Lieberman [43,44], Marcellini [48], Mihăilescu-Pucci-Rădulescu [50], Mihăilescu-RădulescuTersian [51], Namlyeyeva-Shishkov-Skrypnik [52], Skrypnik [61], TersenovTersenov [64], and Vétois [66,68,69].

We give examples of solutions and Palais-Smale sequences for problem (1.2) in Section 2, we discuss our geometric hypothesis on the domain $\Omega$ in Sect. 3, we prove a concentration result in Sect. 4, and we prove Theorem 1.1 in Sect. 5.

\section{Examples of solutions and Palais-Smale sequences}

This section is devoted to the construction of solutions and Palais-Smale sequences for problem (1.2). We begin with providing a class of examples in the situation where $\vec{p}$ consists in two distinct exponents $p_{-}$and $p_{+}$. In other words, we assume in the following that there exist $n_{-} \geq 1$ and $n_{+} \geq 1$ such that $n=n_{-}+n_{+}, p_{1}=\cdots=p_{n_{-}}=p_{-}$, and $p_{n_{-}+1}=\cdots=p_{n}=p_{+}$. Proposition 2.1 below is the basic tool in our constructions. We state our examples in Corollaries 2.2, 2.3, 2.4, and 2.5. Proposition 2.1 relies on a direct computation.

Proposition 2.1. Let $n_{-} \geq 1, n_{+} \geq 1, n=n_{-}+n_{+}$, and $\vec{p}=\left(p_{1}, \ldots, p_{n}\right)$, and assume that $p_{1}=\cdots=p_{n_{-}}=p_{-}$and $p_{n_{-}+1}=\cdots=p_{n}=p_{+}$. Let $\varepsilon$ and $\lambda$ be two real numbers. Let $\Omega=\Omega_{1} \times \Omega_{2}$, where $\Omega_{1}$ is a nonempty, open subset of $\mathbb{R}^{n_{-}}$and $\Omega_{2}$ is a nonempty, open subset of $\mathbb{R}^{n_{+}}$. Let $v$ be a solution of the problem

$$
\left\{\begin{array}{l}
-\sum_{i=1}^{n_{-}} \frac{\partial}{\partial x_{i}}\left(\left|\frac{\partial v}{\partial x_{i}}\right|^{p_{-}-2} \frac{\partial v}{\partial x_{i}}\right)=|v|^{p_{+}-2} v+\varepsilon|v|^{p_{-}-2} v \quad \text { in } \Omega_{1}, \\
v \in D^{1, p_{-}}\left(\Omega_{1}\right) \cap L^{p_{+}}\left(\Omega_{1}\right)\left(\cap L^{p_{-}}\left(\Omega_{1}\right) \quad \text { if } \varepsilon \neq 0 \quad \text { and } \quad \Omega_{1} \text { is unbounded }\right),
\end{array}\right.
$$

and let $w$ be a solution in $D^{1, \vec{p}}\left(\Omega_{2}\right)$ of the problem

$$
\left\{\begin{array}{l}
-\sum_{i=1}^{n_{+}} \frac{\partial}{\partial x_{i}}\left(\left|\frac{\partial w}{\partial x_{i}}\right|^{p_{+}-2} \frac{\partial w}{\partial x_{i}}\right)=\lambda|w|^{p_{+}-2} w-|w|^{p_{-}-2} w \quad \text { in } \Omega_{2}, \\
w \in D^{1, p_{+}}\left(\Omega_{2}\right) \cap L^{p_{-}}\left(\Omega_{2}\right) .
\end{array}\right.
$$

Then the function $u$ defined on $\Omega$ by

$$
u\left(x_{1}, \ldots, x_{n}\right)=v\left(x_{1}, \ldots, x_{n_{-}}\right) w\left(x_{n_{-}+1}, \ldots, x_{n}\right)
$$

is a solution in $D^{1, \vec{p}}(\Omega)$ of the problem

$\left\{\begin{array}{l}-\Delta_{\vec{p}} u=\lambda|u|^{p_{+}-2} u+\varepsilon|u|^{p_{-}-2} u \quad \text { in } \Omega, \\ u \in D^{1, \vec{p}}(\Omega) \cap L^{p_{+}}(\Omega)\left(\cap L^{p_{-}}(\Omega) \quad \text { if } \varepsilon \neq 0 \quad \text { and } \Omega \text { is unbounded }\right) .\end{array}\right.$

Proof. A direct computation provides the result.

In case $\varepsilon=0, \Omega_{1}=\mathbb{R}^{n_{-}}$, and $\Omega_{2}=\mathbb{R}^{n_{+}}$, see Vétois [68], we have the following corollary. 
Corollary 2.2. Let $n_{-} \geq 2, n_{+} \geq 1, n=n_{-}+n_{+}$, and $\vec{p}=\left(p_{1}, \ldots, p_{n}\right)$, and assume that $p_{1}=\cdots=p_{n_{-}}=p_{-}, p_{n_{-}+1}=\cdots=p_{n}=p_{+}$, and $p_{+}=p^{*}$. For any point $a=\left(a_{1}, \ldots, a_{n}\right)$ in $\mathbb{R}^{n}$ and for any positive real numbers $\mu$ and $\lambda$, there exists a nonnegative solution $\mathcal{U}_{a, \mu, \lambda}$ in $D^{1, \vec{p}}\left(\mathbb{R}^{n}\right) \cap C^{1}\left(\mathbb{R}^{n}\right)$ of problem (2.4) with $\varepsilon=0$ and $\Omega=\mathbb{R}^{n}$ of the form

$$
\begin{aligned}
\mathcal{U}_{a, \mu, \lambda}\left(x_{1}, \ldots, x_{n}\right)= & \mu^{-1} \lambda^{\frac{-1}{p_{+}-p_{-}}} \mathcal{U}\left(\mu^{\frac{p_{-}-p_{+}}{p_{-}}}\left(x_{1}-a_{1}\right), \ldots, \mu^{\frac{p_{-}-p_{+}}{p_{-}}}\left(x_{n_{-}}-a_{n_{-}}\right),\right. \\
& \left.\lambda^{\frac{1}{p_{+}}}\left(x_{n_{-}+1}-a_{n_{-}+1}\right), \ldots, \lambda^{\frac{1}{p_{+}}}\left(x_{n}-a_{n}\right)\right),
\end{aligned}
$$

where

$$
\begin{aligned}
\mathcal{U}\left(x_{1}, \ldots, x_{n}\right)= & \left(\frac{n_{-}\left(n_{-}-p_{-}\right)^{p_{-}-1}}{\left(p_{-}-1\right)^{p_{-}-1}}\right)^{\frac{n_{-} p_{-}}{p_{-}^{2}}}\left(\frac{1}{1+\sum_{i=1}^{n_{-}}\left|x_{i}\right|^{\frac{p_{-}}{p_{-}}}}\right)^{\frac{n_{-}-p_{-}}{p_{-}}} \\
& \times \mathcal{W}\left(\left(\sum_{i=n_{-}+1}^{n}\left|x_{i}\right|^{\frac{p_{+}}{p_{+}-1}}\right)^{\frac{p_{+}-1}{p_{+}}}\right),
\end{aligned}
$$

where $\mathcal{W}$ is such that $\mathcal{W}>0$ and $\mathcal{W}^{\prime}<0$ in $(0, R)$, and $\mathcal{W}=0$ in $[R,+\infty)$ for some $R>0$.

Since the function $\mathcal{W}$ has compact support, Corollary 2.2 provides a class of solutions of the purely invariant problem (1.6) on cylindric domains $\Omega=\mathbb{R}^{n_{-}} \times V$ for all nonempty, open subsets $V$ of $\mathbb{R}^{n_{+}}$.

Now, we consider the case of a bounded domain $\Omega=\Omega_{1} \times \Omega_{2}$. Using a result by Franchi-Lanconelli-Serrin [33], we get the existence of a solution of problem (2.2) which has compact support in $\Omega_{2}$ for $\lambda>0$ large (see Vétois [68]). In order to apply Proposition 2.1, it remains to find solutions of problem (2.1).

In case $n_{-} \geq 3, p_{-}=2$, and $p_{+}=2^{*}$, see Bahri-Coron [9], we get the existence of a nonnegative, nontrivial solution of problem (2.1) with $\varepsilon=0$ for noncontractible, smooth, bounded domains $\Omega_{1}$. Therefore, we state a second corollary of Proposition 2.1 as follows.

Corollary 2.3. Let $n_{-} \geq 3, n_{+} \geq 1, n=n_{-}+n_{+}$, and $\vec{p}=\left(p_{1}, \ldots, p_{n}\right)$, and assume that $p_{1}=\cdots=p_{n_{-}}=2, p_{n_{-}+1}=\cdots=p_{n}=2^{*}$. Let $\Omega=\Omega_{1} \times \Omega_{2}$, where $\Omega_{1}$ is a nonempty, noncontractible, smooth, bounded, open subset of $\mathbb{R}^{n_{-}}$ and $\Omega_{2}$ is a nonempty, open subset of $\mathbb{R}^{n_{+}}$. Given a point $a=\left(a_{1}, \ldots, a_{n}\right)$ in $\Omega_{2}$, for $\lambda>0$ large, there exists a nonnegative, nontrivial solution of the form (2.3), in $D^{1, \vec{p}}(\Omega) \cap C^{1}(\Omega)$, of problem (2.4) with $\varepsilon=0$. 
In case $n_{-} \geq 4, p_{-}=2$, and $p_{+}=2^{*}$, see Brézis-Nirenberg [19], we get the existence of a nonnegative, nontrivial solution of problem (2.1) for $\varepsilon>0$ small, on smooth, bounded domains $\Omega_{1}$. We then state a third corollary of Proposition 2.1 as follows.

Corollary 2.4. Let $n_{-} \geq 4, n_{+} \geq 1, n=n_{-}+n_{+}$, and $\vec{p}=\left(p_{1}, \ldots, p_{n}\right)$, and assume that $p_{1}=\cdots=p_{n_{-}}=2, p_{n_{-}+1}=\cdots=p_{n}=2^{*}$. Let $\Omega=\Omega_{1} \times \Omega_{2}$, where $\Omega_{1}$ is a nonempty, smooth, bounded, open subset of $\mathbb{R}^{n_{-}}$and $\Omega_{2}$ is a nonempty, open subset of $\mathbb{R}^{n_{+}}$. Given a point $a=\left(a_{1}, \ldots, a_{n}\right)$ in $\Omega_{2}$, for $\lambda>0$ large and $\varepsilon>0$ small, there exists a nonnegative, nontrivial solution of the form (2.3), in $D^{1, \vec{p}}(\Omega) \cap C^{1}(\Omega)$, of the problem (2.4).

In case $n_{-} \geq 5$, see Rey $[56,57]$, we get the existence of families of solutions of problem $(2.1)$ with $0<\varepsilon \ll 1$ on smooth, bounded domains $\Omega_{1}$, which are bounded in $D^{1,2}\left(\Omega_{1}\right)$ and which blow up as $\varepsilon \rightarrow 0$ at nondegenerate, critical points of the regular part of Green's function. Using this result, we state a fourth corollary of Proposition 2.1 as follows.

Corollary 2.5. Let $n_{-} \geq 5, n_{+} \geq 1, n=n_{-}+n_{+}$, and $\vec{p}=\left(p_{1}, \ldots, p_{n}\right)$, and assume that $p_{1}=\cdots=p_{n_{-}}=2, p_{n_{-}+1}=\cdots=p_{n}=2^{*}$. Let $\Omega=\Omega_{1} \times \Omega_{2}$, where $\Omega_{1}$ is a nonempty, smooth, bounded, open subset of $\mathbb{R}^{n_{-}}$and $\Omega_{2}$ is a nonempty, open subset of $\mathbb{R}^{n_{+}}$. Let $\left(\varepsilon_{\alpha}\right)_{\alpha}$ be a sequence of positive real numbers converging to 0 . Given a point $a=\left(a_{1}, \ldots, a_{n}\right)$ in $\Omega_{2}$, for $\lambda>0$ large, there exists a bounded, blowing up, Palais-Smale sequence for the functional $I_{\infty}$ defined in (1.10) with $f \equiv 0$.

We end this section with a more classical example of Palais-Smale sequences which holds in the general situation. We let $n \geq 3$ and $\vec{p}=\left(p_{1}, \ldots, p_{n}\right)$, and we assume that $\sum_{i=1}^{n} 1 / p_{i}>1$ and $p_{+}=p^{*}$. We let $\lambda$ be a positive real number, $\Omega$ be a bounded, open subset of $\mathbb{R}^{n}$, and $f$ be a Caratheodory function in $\Omega \times \mathbb{R}$ satisfying the growth condition (1.3). We let $\left(r_{\alpha}\right)_{\alpha}$ be a sequence of real numbers converging to $p_{+}$, and we assume that $q<r_{\alpha}<p_{+}$for all $\alpha$, where $q$ is the real number in (1.3). By standard variational arguments, for any $\alpha$, we then get the existence of a function $u_{\alpha}$ in $D^{1, \vec{p}}(\Omega)$ such that there hold $\int_{\Omega}\left|u_{\alpha}\right|^{r_{\alpha}} d x=\lambda$ and

$$
\begin{aligned}
& \sum_{i=1}^{n} \frac{1}{p_{i}} \int_{\Omega}\left|\frac{\partial u_{\alpha}}{\partial x_{i}}\right|^{p_{i}} d x-\int_{\Omega} F\left(x, u_{\alpha}\right) d x \\
& =\min _{\substack{u \in D^{1, \vec{p}}(\Omega) \\
\int_{\Omega}|u|^{r_{\alpha}} d x=\lambda}}\left(\sum_{i=1}^{n} \frac{1}{p_{i}} \int_{\Omega}\left|\frac{\partial u}{\partial x_{i}}\right|^{p_{i}} d x-\int_{\Omega} F(x, u) d x\right)
\end{aligned}
$$

where $F(x, u)=\int_{0}^{u} f(x, s) d s$. In particular, for any $\alpha$, there exists a positive real number $\lambda_{\alpha}$ such that the function $u_{\alpha}$ is a solution of problem (1.8). The sequence $\left(u_{\alpha}\right)_{\alpha}$ is a bounded Palais-Smale sequence for the functionals $\left(I_{\alpha}\right)_{\alpha}$ defined in (1.9). 


\section{The asymptotic stability of domains}

This section is devoted to the geometric condition of asymptotic stability of domains which is in the statement of Theorem 1.1. The notion of asymptotically stable domains was introduced in El Hamidi-Vétois [29]. We state the definition below, and we comment this notion in our critical setting $p_{+}=p^{*}$. We first recall that a nonempty subset $U$ of $\mathbb{R}^{n}$ is said to satisfy the segment prop$e r t y$ if for any point $a$ on $\partial U$, there exist a neighborhood $X_{a}$ of $a$ and a nonzero vector $\sigma_{a}$ such that $X_{a} \cap \bar{U}+t \sigma_{a} \subset U$ for all real numbers $t$ in $(0,1)$. Adapting classical arguments, as developed, for instance, in Adams-Fournier [1], we get that any nonempty, open subset $U$ of $\mathbb{R}^{n}$ satisfying the segment property is such that the anisotropic Sobolev space $D^{1, \vec{p}}(U)$ consists of the restrictions to $U$ of functions in $D^{1, \vec{p}}\left(\mathbb{R}^{n}\right)$ with support in $\bar{U}$. The definition of asymptotically stable domains states as follows.

Definition 3.1. A nonempty, open subset $\Omega$ of $\mathbb{R}^{n}$ is said to be asymptotically $\vec{p}$-stable if for any sequence $\left(\mu_{\alpha}\right)_{\alpha}$ of positive real numbers converging to 0 and for any sequence $\left(x_{\alpha}\right)_{\alpha}$ in $\mathbb{R}^{n}$, the sets $\Omega_{\alpha}=\tau_{\mu_{\alpha}, x_{\alpha}}^{\vec{p}}(\Omega)$, where $\tau_{\mu_{\alpha}, x_{\alpha}}^{\vec{p}}$ is as in (1.5), converge, up to a subsequence, as $\alpha \rightarrow+\infty$, to an open subset $U$ of $\mathbb{R}^{n}$ which is either empty or satisfies the segment property. The convergence is in the sense that the two following properties hold true:

(i) any compact subset of $U$ is included in $\Omega_{\alpha}$ for $\alpha$ large,

(ii) for any compact $K \subset \mathbb{R}^{n}$, there holds $\left|K \cap \Omega_{\alpha} \backslash U\right| \rightarrow 0$ as $\alpha \rightarrow+\infty$.

We use the notation $\Omega_{\alpha} \rightarrow U$ when (i)-(ii) are satisfied.

Limits in the sense of (i)-(ii) are unique up to sets of measure zero. Uniqueness, without subtracting sets of measure zero, is recovered when requiring in addition that the limit set is open and satisfies the segment property. The asymptotic stability of domains is a subtle notion. Figure 2 below illustrates what can go wrong with a domain which is not asymptotically stable. The annular cylinder in Fig. 2 bends on itself and converges to a domain of the type $\left(\mathbb{R}^{2} \backslash \mathcal{H}\right) \times[a, b]$, where $a<b$ and $\mathcal{H}$ is a halfline. In particular, the limit domain does not satisfy the segment property, and thus the annular cylinder is not asymptotically stable.

On the other hand, the ball, illustrated in Fig. 1, provides an example of a domain which is asymptotically $\vec{p}$-stable for any anisotropic configuration $\vec{p}$. More generally we get the following result.

Theorem 3.2. Let $n \geq 3$ and $\vec{p}=\left(p_{1}, \ldots, p_{n}\right)$, and assume that $\sum_{i=1}^{n} 1 / p_{i}>1$ and $p_{+}=p^{*}$. Then any nonempty, convex, bounded, open subset of $\mathbb{R}^{n}$ is asymptotically $\vec{p}$-stable. Moreover, the limit domains $U$ in Definition 3.1 can be chosen to be either empty or convex.

Proof. We refer to Vétois [67, Theorem 2.1] for the proof of Theorem 3.2 which works the same as in case $p_{+}<p^{*}$.

The convexity provides an easy geometric condition for asymptotic stability of domains. Contrary to the case $p_{+}<p^{*}$, there is no regularity type condition. Indeed, there exist domains which are not asymptotically stable for 


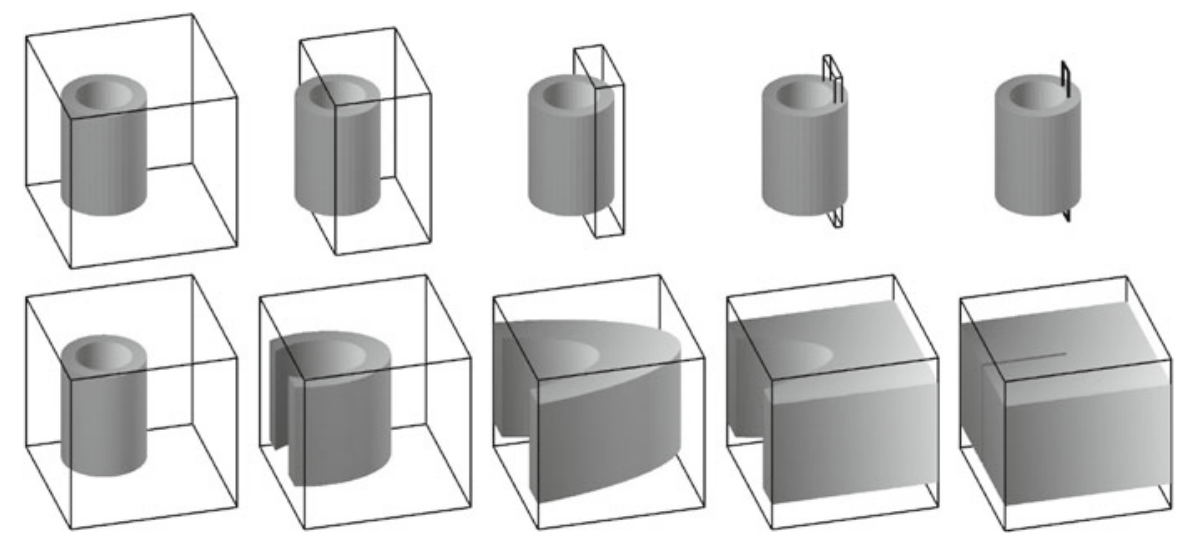

FiguRE 2. Rescaling of an annular cylinder $\left(n=3, p_{1}=1.1\right.$, $\left.p_{2}=2, p_{3}=44 / 9\right)$. The first line describes the scale in the rescaling. The second line describes the deformation of the domain

all anisotropic configurations satisfying $p_{+}=p^{*}$. For instance, we can easily show that annular domains of the type $\mathcal{A}=\left\{x \in \mathbb{R}^{n} ; \quad a<|x|<b\right\}$, where $0<a<b$, are not asymptotically stable in case $p_{+}=p^{*}$. In order to prove this claim, we let $I_{+}=\left\{i \in\{1, \ldots, n\} ; \quad p_{i}=p_{+}\right\}, i_{0}$ be such that $p_{i_{0}} \neq p_{+}$, $x_{0}=\left(x_{1}^{0}, \ldots, x_{n}^{0}\right)$ be the point in $\mathbb{R}^{n}$ such that $x_{n}^{i_{0}}=a$ and $x_{n}^{i}=0$ for all $i \neq i_{0}$, and $\left(\mu_{\alpha}\right)_{\alpha}$ be a sequence of positive real numbers converging to 0 . We then get that $\tau_{\mu_{\alpha}, x_{0}}^{\vec{p}}(\mathcal{A}) \rightarrow\left\{x \in \mathbb{R}^{n} ; 0<\sum_{i \in I_{+}} x_{i}^{2}<b^{2}-a^{2}\right\}$ as $\alpha \rightarrow+\infty$, and that the limit domain does not satisfy the segment property. It follows that the domain $\mathcal{A}$, even though being smooth, is not asymptotically stable in case $p_{+}=p^{*}$.

In Theorem 3.3 below, we give a general class of asymptotically stable domains as products of two domains, one of which being asymptotically stable with respect to noncritical directions, and the other satisfying the segment property in critical directions. One can find a detailed discussion in El HamidiVétois [29] and Vétois [67] about asymptotically stable domains with respect to noncritical directions. Our result states as follows.

Theorem 3.3. Let $n \geq 3, \vec{p}=\left(p_{1}, \ldots, p_{n}\right)$, and assume that $\sum_{i=1}^{n} 1 / p_{i}>1$, $p_{+}=p^{*}, p_{n-n_{+}+1}=\cdots=p_{n}=p_{+}$, and $p_{i}<p_{+}$for all $i \leq n-n_{+}$. Let $\Omega_{1}$ be a nonempty, bounded, open subset of $\mathbb{R}^{n-n_{+}}$and $\Omega_{2}$ be a nonempty, bounded, open subset of $\mathbb{R}^{n_{+}}$. Then $\Omega_{1} \times \Omega_{2}$ is asymptotically $\vec{p}$-stable if and only if $\Omega_{1}$ is asymptotically $\vec{q}$-stable, where $\vec{q}=\left(p_{1}, \ldots, p_{n-n_{+}}\right)$, and $\Omega_{2}$ satisfies the segment property.

Proof of Theorem 3.3. We let $\left(\mu_{\alpha}\right)_{\alpha}$ be a sequence of positive real numbers converging to $0,\left(x_{\alpha}^{1}\right)_{\alpha}$ be a sequence of points in $\mathbb{R}^{n-n_{+}}$, and $\left(x_{\alpha}^{2}\right)_{\alpha}$ be a sequence of points in $\mathbb{R}^{n_{+}}$. For any $\alpha$, we let $x_{\alpha}=\left(x_{\alpha}^{1}, x_{\alpha}^{2}\right)$. We remark that

$$
\tau_{\mu_{\alpha}, x_{\alpha}}^{\vec{p}}\left(\Omega_{1} \times \Omega_{2}\right)=\tau_{\mu_{\alpha}, x_{\alpha}^{1}}^{\vec{q}}\left(\Omega_{1}\right) \times\left(\Omega_{2}-x_{\alpha}^{2}\right),
$$


where $\vec{p}=\left(p_{1}, \ldots, p_{n}\right)$ and $\vec{q}=\left(p_{1}, \ldots, p_{n-n_{+}}\right)$. We first assume that $\Omega_{1}$ is asymptotically $\vec{q}$-stable, and that $\Omega_{2}$ satisfies the segment property. Passing if necessary to a subsequence, we get that there exists an open subset $U_{1}$ of $\mathbb{R}^{n-n_{+}}$, either empty or satisfying the segment property, such that $\tau_{\mu_{\alpha}, x_{\alpha}^{1}}^{\vec{q}}\left(\Omega_{1}\right) \rightarrow U_{1}$ as $\alpha \rightarrow+\infty$ in the sense of Definition 3.1. In case $\left|x_{\alpha}^{2}\right| \rightarrow$ $+\infty$ as $\alpha \rightarrow+\infty$, we easily get that $\tau_{\mu_{\alpha}, x_{\alpha}}^{\vec{p}}\left(\Omega_{1} \times \Omega_{2}\right) \rightarrow \emptyset$ as $\alpha \rightarrow+\infty$. Therefore, passing if necessary to another subsequence, we may assume that the sequence $\left(x_{\alpha}^{2}\right)_{\alpha}$ converges to a point $a_{2}$ in $\mathbb{R}^{n_{+}}$. We then claim that $\Omega_{2}-x_{\alpha}^{2} \rightarrow \Omega_{2}-a_{2}$ as $\alpha \rightarrow+\infty$, or equivalently that $\Omega_{2}-z_{\alpha} \rightarrow \Omega_{2}$ as $\alpha \rightarrow+\infty$, where $z_{\alpha}=x_{\alpha}^{2}-a_{2}$. For any compact subset $K$ of $\Omega_{2}$, we get $d\left(K, \mathbb{R}^{n} \backslash\left(\Omega_{2}-z_{\alpha}\right)\right) \geq d\left(K, \mathbb{R}^{n} \backslash \Omega_{2}\right)-\left|z_{\alpha}\right|$ for all $\alpha$. Since $z_{\alpha} \rightarrow 0$ as $\alpha \rightarrow+\infty$, it follows that $\liminf \operatorname{in}_{\alpha \rightarrow+\infty} d\left(K, \mathbb{R}^{n} \backslash\left(\Omega_{2}-z_{\alpha}\right)\right)>0$, and thus that $K \subset \Omega_{2}-z_{\alpha}$ for $\alpha$ large. Now, we prove that for any compact subset $K$ of $\mathbb{R}^{n_{+}}$, there holds $\left|K \cap\left(\Omega_{2}-z_{\alpha}\right) \backslash \Omega_{2}\right| \rightarrow 0$ as $\alpha \rightarrow+\infty$. As is easily seen, it suffices to show that for any point $a$ in $\mathbb{R}^{n_{+}}$, there exists an open neighborhood $X_{a}$ of $a$ such that $\left|X_{a} \cap\left(\Omega_{2}-z_{\alpha}\right) \backslash \Omega_{2}\right| \rightarrow 0$ as $\alpha \rightarrow+\infty$. In case $a$ belongs to $\Omega_{2}$, for $r>0$ small, there holds $B_{a}(r) \subset \Omega_{2}$, and thus $\left|B_{a}(r) \cap\left(\Omega_{2}-z_{\alpha}\right) \backslash \Omega_{2}\right|=0$ for all $\alpha$. In case $a$ belongs to $\mathbb{R}^{n_{+}} \backslash \bar{\Omega}_{2}$, for $r>0$ small, there holds $\overline{B_{a}(r)} \subset \mathbb{R}^{n_{+}} \backslash \bar{\Omega}_{2}$, $B_{a}(r) \subset \mathbb{R}^{n_{+}} \backslash\left(\Omega_{2}-z_{\alpha}\right)$ for $\alpha$ large, and thus $\left|B_{a}(r) \cap\left(\Omega_{2}-z_{\alpha}\right) \backslash \Omega_{2}\right|=0$ for $\alpha$ large. Now, we assume that the point $a$ belongs to the boundary of $\Omega_{2}$. Since $\Omega_{2}$ satisfies the segment property, by Maz'ya-Poborchi [49, Section 1.3.1], we get that there exists a Cartesian coordinate system $\left(\xi_{1}, \ldots, \xi_{n_{+}}\right)$of $\mathbb{R}^{n_{+}}$, a continuous function $\varphi_{a}: \mathbb{R}^{n_{+}-1} \rightarrow \mathbb{R}$, and an open neighborhood $X_{a}$ of $a$ such that the set $X_{a} \cap \Omega_{2}$ consists of the points $\left(\xi_{1}, \ldots, \xi_{n_{+}}\right)$in $X_{a}$ satisfying $\xi_{n_{+}}<\varphi_{a}\left(\xi_{1}, \ldots, \xi_{n_{+}-1}\right)$. We then let $Y_{a}$ be a bounded, open neighborhood of $a$ such that $\overline{Y_{a}} \subset X_{a}$. Since $z_{\alpha} \rightarrow 0$ as $\alpha \rightarrow+\infty$, it follows that, for $\alpha$ large, the set $Y_{a} \cap\left(\Omega_{2}-z_{\alpha}\right) \backslash \Omega_{2}$ consists of the points $\left(\xi_{1}, \ldots, \xi_{n_{+}}\right)$in $Y_{a}$ satisfying $\varphi_{a}\left(\xi_{1}, \ldots, \xi_{n_{+}-1}\right) \leq \xi_{n_{+}}<\varphi_{a}\left(\xi_{1}+z_{1}^{\alpha}, \ldots, \xi_{n_{+}-1}+z_{n_{+}-1}^{\alpha}\right)-z_{n_{+}}^{\alpha}$, where $z_{\alpha}=\left(z_{1}^{\alpha}, \ldots, z_{n_{+}}^{\alpha}\right)$ in the coordinate system $\left(\xi_{1}, \ldots, \xi_{n_{+}}\right)$. By the continuity of the function $\varphi_{a}$, we then get $\left|Y_{a} \cap\left(\Omega_{2}-z_{\alpha}\right) \backslash \Omega_{2}\right| \rightarrow 0$ as $\alpha \rightarrow+\infty$. This ends the proof of our claim, namely that $\Omega_{2}-x_{\alpha}^{2} \rightarrow \Omega_{2}-a_{2}$ as $\alpha \rightarrow+\infty$. Taking into account (3.1), we then get $\tau_{\mu_{\alpha}, x_{\alpha}}^{\vec{p}}\left(\Omega_{1} \times \Omega_{2}\right) \rightarrow U_{1} \times\left(\Omega_{2}-a_{2}\right)$ as $\alpha \rightarrow+\infty$. Moreover, in case $U_{1}$ is nonempty, since both $U_{1}$ and $\Omega_{2}$ satisfy the segment property, we get that $U_{1} \times\left(\Omega_{2}-a_{2}\right)$ satisfies the segment property. Since this holds true for all sequences $\left(\mu_{\alpha}\right)_{\alpha}$ and $\left(x_{\alpha}\right)_{\alpha}$, it follows that $\Omega_{1}$ is asymptotically $\vec{p}$-stable. Conversely, we assume that $\Omega_{1} \times \Omega_{2}$ is asymptotically $\vec{p}$-stable. For any $\alpha$, we let $x_{\alpha}=\left(x_{\alpha}^{1}, x_{\alpha}^{2}\right)$, where $x_{\alpha}^{2}=0$ is fixed. Passing if necessary to a subsequence, we get that there exists an open subset $U$ of $\mathbb{R}^{n}$, either empty or satisfying the segment property, such that $\tau_{\mu_{\alpha}, x_{\alpha}}^{\vec{p}}\left(\Omega_{1} \times \Omega_{2}\right) \rightarrow U$ as $\alpha \rightarrow+\infty$. We let $U_{1}=\left\{x \in \mathbb{R}^{n-n_{+}} ; \quad \exists y \in \Omega_{2} \quad(x, y) \in U\right\}$. We claim that $U=U_{1} \times \Omega_{2}$. Clearly, we get $U \subset U_{1} \times \Omega_{2}$. On the other hand, we proceed by contradiction and assume that there exists a point $\left(x_{1}, x_{2}\right)$ in $U_{1} \times \Omega_{2}$ which does not belong to $U$. By definition of $U_{1}$, there exists a point $y_{2}$ in $\Omega_{2}$ such that $\left(x_{1}, y_{2}\right)$ belongs to $U$. It follows that $\overline{B_{x_{1}}(\varepsilon) \times B_{y_{2}}(\varepsilon)} \subset U$ for small positive real numbers $\varepsilon$. Moreover, since $\tau_{\mu_{\alpha}, x_{\alpha}}^{\vec{p}}(\Omega) \rightarrow U$, we then get $\overline{B_{x_{1}}(\varepsilon)} \subset \tau_{\mu_{\alpha}, x_{\alpha}^{1}}^{\vec{q}}\left(\Omega_{1}\right)$ for 

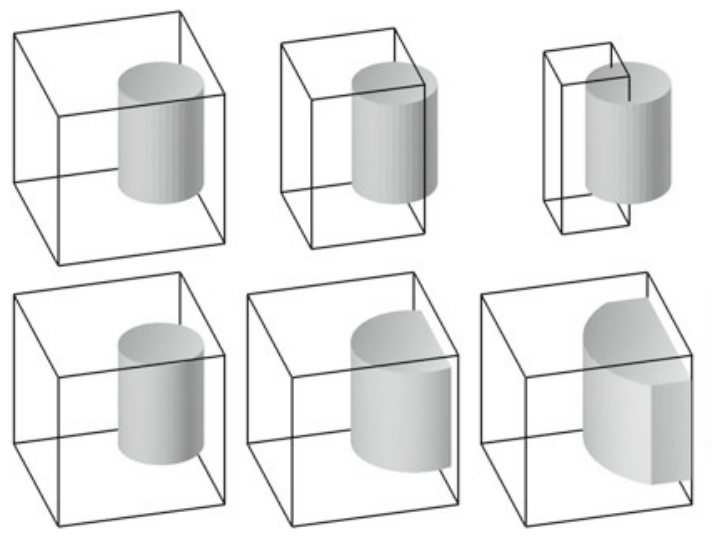
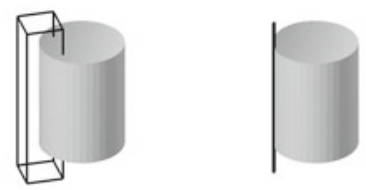

Figure 3. Rescaling of a cylinder $\left(n=3, p_{1}=p_{2}=1.5\right.$, $\left.p_{3}=6\right)$. The first line describes the scale in the rescaling. The second line describes the deformation of the domain
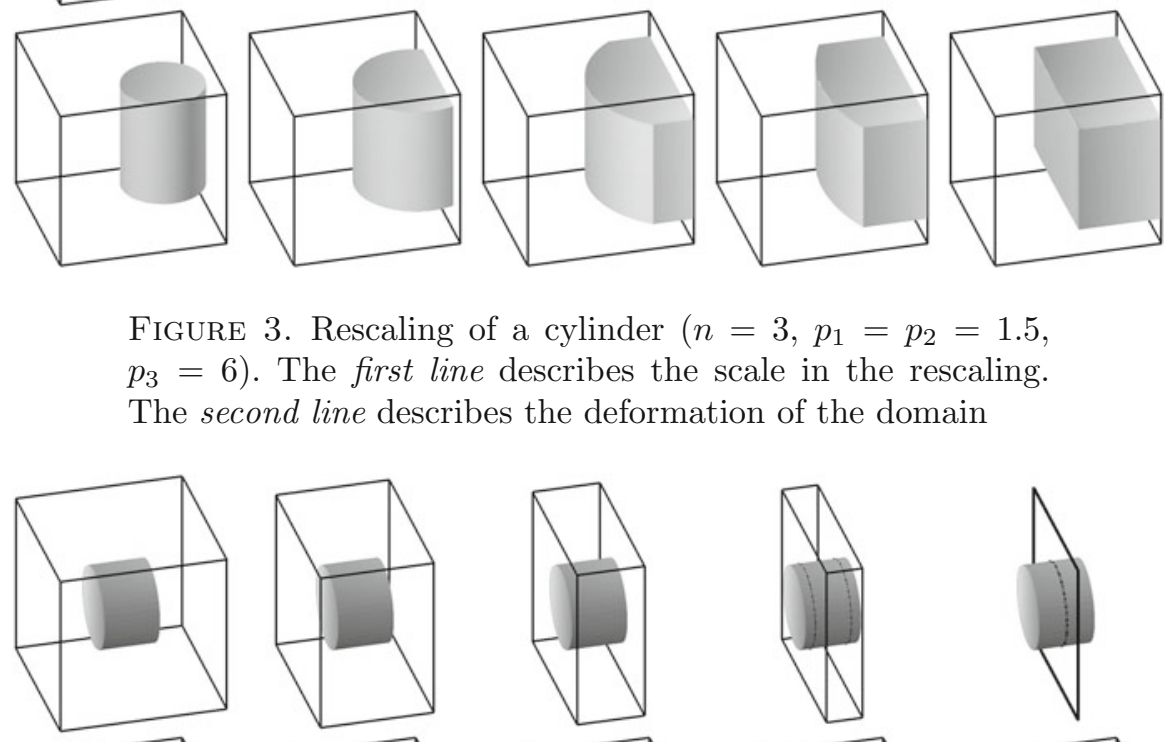
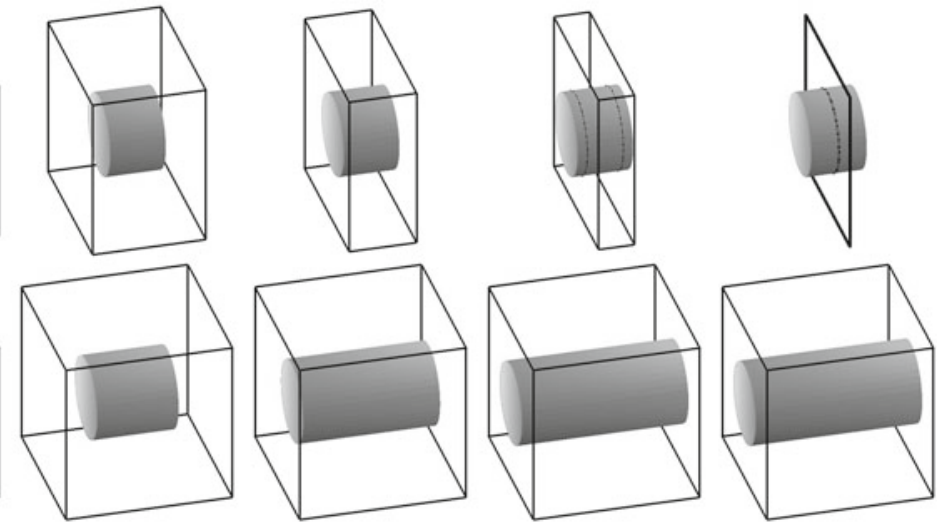

FiguRE 4. Rescaling of $(0,1)^{2} \times \mathbb{B}^{2}\left(n=4, p_{1}=p_{2}=1.5\right.$, $p_{3}=p_{4}=6$, projection onto $\left.\{0\} \times \mathbb{R}^{3}\right)$. The first line describes the scale in the rescaling. The second line describes the deformation of the domain

$\alpha$ large, and we also get $\left|\left(B_{x_{1}}(\varepsilon) \times B_{x_{2}}(\varepsilon)\right) \backslash U\right|=0$. This is in contradiction with the segment property satisfied by $U$. This ends the proof of our claim, namely that $U=U_{1} \times \Omega_{2}$. By $(3.1)$ and since $\tau_{\mu_{\alpha}, x_{\alpha}}^{\vec{p}}(\Omega) \rightarrow U$, it follows that $\tau_{\mu_{\alpha}, x_{\alpha}^{1}}^{\vec{q}}\left(\Omega_{1}\right) \rightarrow U_{1}$ as $\alpha \rightarrow+\infty$. Moreover, since $U$ satisfies the segment property, we also get that both $U_{1}$ and $\Omega_{2}$ satisfy the segment property. Since this holds true for all sequences $\left(\mu_{\alpha}\right)_{\alpha}$ and $\left(x_{\alpha}^{1}\right)_{\alpha}$, it follows that $\Omega_{1}$ is asymptotically $\vec{q}$-stable. This ends the proof of Theorem 3.3 .

Figures 3 and 4 above illustrate Theorem 3.3 in the cases of two different cylindric domains. In Fig. 3 , we rescale the cylinder $\mathbb{B}^{2} \times(0,1)$, where $\mathbb{B}^{2}$ is the 
unit two-dimensional ball. The limit domain is of the form $\mathcal{H} \times(0,1)$, where $\mathcal{H}$ is a halfplane. In Fig. 4, we rescale the cylinder $(0,1)^{2} \times \mathbb{B}^{2}$ (in projection onto $\{0\} \times \mathbb{R}^{3}$ ). The limit domain is $\mathbb{R}^{2} \times \mathbb{B}^{2}$.

\section{The concentration on critical directions}

In this section, we prove a concentration result which is the main tool in the proof of Theorem 1.1. We let $\left(\Omega_{\alpha}\right)_{\alpha}$ be a sequence of nonempty, bounded, open subsets of $\mathbb{R}^{n},\left(\lambda_{\alpha}\right)_{\alpha}$ be a sequence of positive real numbers, and $\left(r_{\alpha}\right)_{\alpha}$ be a sequence of real numbers in $\left(1, p_{+}\right]$converging to $p_{+}$. For any $\alpha$, we define the functional $\left(J_{\alpha}\right)_{\alpha}$ by

$$
J_{\alpha}(u)=\sum_{i=1}^{n} \frac{1}{p_{i}} \int_{\Omega_{\alpha}}\left|\frac{\partial u}{\partial x_{i}}\right|^{p_{i}} d x-\frac{\lambda_{\alpha}}{r_{\alpha}} \int_{\Omega_{\alpha}}|u|^{r_{\alpha}} d x
$$

for all functions $u$ in $D^{1, \vec{p}}\left(\Omega_{\alpha}\right)$. We say that a sequence $\left(u_{\alpha}\right)_{\alpha}$ is Palais-Smale for the functionals $\left(J_{\alpha}\right)_{\alpha}$ if there hold $u_{\alpha} \in D^{1, \vec{p}}\left(\Omega_{\alpha}\right),\left|J_{\alpha}\left(u_{\alpha}\right)\right| \leq C$ for some positive constant $C$ independent of $\alpha$, and $\left\|D J_{\alpha}\left(u_{\alpha}\right)\right\|_{D^{1, \vec{p}}\left(\Omega_{\alpha}\right)^{\prime}} \rightarrow 0$ as $\alpha \rightarrow+\infty$. Moreover, $\left(u_{\alpha}\right)_{\alpha}$ is said to be bounded if there hold

$$
\lambda_{\alpha} \int_{\Omega_{\alpha}}\left|u_{\alpha}\right|^{r_{\alpha}} d x \leq C \quad \text { and } \quad \lambda_{\alpha}^{\frac{p_{+}}{p_{+}-1}} \int_{\Omega_{\alpha}}\left|u_{\alpha}\right|^{\frac{p_{+}\left(r_{\alpha}-1\right)}{p_{+}-1}} d x \leq C
$$

for some positive constant $C$ independent of $\alpha$. In particular, bounded Palais-Smale sequences for the functionals $\left(J_{\alpha}\right)_{\alpha}$ are bounded in $D^{1, \vec{p}}\left(\mathbb{R}^{n}\right)$. Our concentration result states as follows.

Proposition 4.1. Let $n \geq 3$ and $\vec{p}=\left(p_{1}, \ldots, p_{n}\right)$, and assume that $\sum_{i=1}^{n} 1 / p_{i}>1, p_{+}=p^{*}, p_{n-n_{+}+1}=\cdots=p_{n}=p_{+}$, and $p_{i}<p_{+}$for all $i \leq n-n_{+}$. Let $U$ be an open subset of $\mathbb{R}^{n}$ either empty or satisfying the segment property, and $\left(\Omega_{\alpha}\right)_{\alpha}$ be a sequence of nonempty, bounded, open subsets of $\mathbb{R}^{n}$ converging to $U$ in the sense of Definition 3.1. Assume that there exists a bounded, open subset $V$ of $\mathbb{R}^{n_{+}}$such that the sets $U$ and $\Omega_{\alpha}$ are included in $\mathbb{R}^{n-n_{+}} \times V$ for all $\alpha$. Let $\left(\lambda_{\alpha}\right)_{\alpha}$ be a sequence of positive real numbers converging to $\lambda \geq 0$, and $\left(r_{\alpha}\right)_{\alpha}$ be a sequence of real numbers in $\left(1, p_{+}\right]$converging to $p_{+}$. Let $\left(u_{\alpha}\right)_{\alpha}$ be a bounded Palais-Smale sequence for the functionals $\left(J_{\alpha}\right)_{\alpha}$ defined in (4.1). Then there exists a function $u_{\infty}$ in $D^{1, \vec{p}}(U)\left(u_{\infty}=0\right.$ in case $U=\emptyset)$ and a finite number $k$ of distinct points $y_{j}=\left(y_{1}^{j}, \ldots, y_{n-n_{+}}^{j}\right)$ in $\mathbb{R}^{n-n_{+}}$, $j=1, \ldots, k$, and nonnegative, nontrivial, finite measures $\xi_{j}$ with support in $\bar{V}_{y_{j}}, j=1, \ldots, k$, where

$$
\bar{V}_{y_{j}}=\left\{\left(y_{1}^{j}, \ldots, y_{n-n_{+}}^{j}\right)\right\} \times \bar{V}
$$

such that, up to a subsequence, there holds

$$
\left|u_{\alpha}\right|^{p_{+}} \longrightarrow\left|u_{\infty}\right|^{p_{+}}+\sum_{j=1}^{k} \xi_{j}
$$


as $\alpha \rightarrow+\infty$ in the sense of measures on compact subsets of $\mathbb{R}^{n}$. Moreover, there exists a positive constant $\Lambda_{0}=\Lambda_{0}(\vec{p})$ such that for any $j=1, \ldots, k$, there holds

$$
\lambda^{\frac{n+p_{+}}{p_{+}}} \xi_{j}\left(\bar{V}_{y_{j}}\right) \geq \Lambda_{0}
$$

where $\bar{V}_{y_{j}}$ is as in (4.3). In particular, if $\lambda=0$, then $k=0$.

Proof of Proposition 4.1. Passing if necessary to a subsequence, we may assume that the sequence $\left(u_{\alpha}\right)_{\alpha}$ converges weakly to a function $u_{\infty}$ in $D^{1, \vec{p}}$ $\left(\mathbb{R}^{n}\right)$ and that $\left(u_{\alpha}\right)_{\alpha}$ converges to $u_{\infty}$ almost everywhere in $\mathbb{R}^{n}$. Since $\Omega_{\alpha} \rightarrow U$ as $\alpha \rightarrow+\infty$ in the sense of Definition 3.1, we get that the support of the function $u_{\infty}$ is included in $\bar{U}$. In case $U \neq \emptyset$, since $U$ satisfies the segment property, it follows that the function $u_{\infty}$ belongs to the anisotropic Sobolev space $D^{1, \vec{p}}(U)$. We fix a positive real number $R$, and we let $B_{0}(R)$ be the $\left(n-n_{+}\right)$dimensional ball of center 0 and radius $R$. For any $\alpha$, we let $v_{\alpha}=u_{\alpha}-u_{\infty}$. By Banach-Alaoglu theorem, since the sequence $\left(v_{\alpha}\right)_{\alpha}$ is bounded in $D^{1, \vec{p}}(U)$ and since the sets $U$ and $\Omega_{\alpha}$ are included in $\mathbb{R}^{n-n_{+}} \times V$, where $V$ is bounded, passing if necessary to a subsequence, we may assume that there exist nonnegative, finite measures $\mu$ and $\nu_{1}, \ldots, \nu_{n}$ on $\overline{B_{0}(2 R)} \times \mathbb{R}^{n_{+}}$such that $\left|v_{\alpha}\right|^{p_{+}} \rightarrow \mu$ and $\left|\partial v_{\alpha} / \partial x_{i}\right|^{p_{i}} \rightarrow \nu_{i}$ as $\alpha \rightarrow+\infty$ in the sense of measures on $\overline{B_{0}(2 R)} \times \mathbb{R}^{n_{+}}$, for all $i=1, \ldots, n$. Moreover, the supports of the measures $\mu$ and $\nu_{1}, \ldots, \nu_{n}$ are included in $\overline{B_{0}(2 R) \times V}$. Since the sequence $\left(u_{\alpha}\right)_{\alpha}$ is bounded in $L^{p_{+}}\left(\mathbb{R}^{n}\right)$ and converges to $u_{\infty}$ almost everywhere in $\mathbb{R}^{n}$, by Brezis-Lieb [18], we get

$$
\int_{U} u_{\alpha}^{p_{+}} \varphi d x \longrightarrow \int_{U} u_{\infty}^{p_{+}} \varphi d x+\int_{B_{0}(2 R) \times V} \varphi d \mu
$$

as $\alpha \rightarrow+\infty$ for all bounded, measurable functions $\varphi$ on $\overline{B_{0}(2 R)} \times \mathbb{R}^{n_{+}}$. Since there holds $\left|\partial u_{\alpha} / \partial x_{i}\right|^{p_{i}} \geq\left|\partial v_{\alpha} / \partial x_{i}\right|^{p_{i}}-\left|\partial u_{\infty} / \partial x_{i}\right|^{p_{i}}$, where $v_{\alpha}=u_{\alpha}-u_{\infty}$ for all $\alpha$ and $i=1, \ldots, n$, we also get

$$
\liminf _{\alpha \rightarrow+\infty} \int_{U}\left|\frac{\partial u_{\alpha}}{\partial x_{i}}\right|^{p_{i}} \varphi d x \geq \int_{\frac{B_{0}(2 R) \times V}{}} \varphi d \nu_{i}-\int_{U}\left|\frac{\partial u_{\infty}}{\partial x_{i}}\right|^{p_{i}} \varphi d x
$$

as $\alpha \rightarrow+\infty$ for all bounded, measurable functions $\varphi$ on $\overline{B_{0}(2 R)} \times \mathbb{R}^{n_{+}}$. Borrowing some ideas in Lions $[45,46]$ with the tricky difference here that the concentration holds on $n_{+}$-dimensional affine subspaces of $\mathbb{R}^{n}$, see the analysis in Vétois [68], we can prove that there exists an at most countable index set $J$ of distinct points $y_{j}$ in $\overline{B_{0}(R)}, j \in J$, such that

$$
\operatorname{Supp} \mu \cap \overline{B_{0}(R) \times V} \subset \bigcup_{j \in J} \bar{V}_{y_{j}},
$$

where $\bar{V}_{y_{j}}$ is as in (4.3). Moreover, since $p_{+}=p^{*}$, by the anisotropic Sobolev inequality in Troisi [65], we get that for any Borelian set $A$ in $\overline{B_{0}(R)}$, there holds 


$$
\mu(A \times \bar{V}) \leq \Lambda \prod_{i=1}^{n} \nu_{i}(A \times \bar{V})^{\frac{p_{+}}{n p_{i}}}
$$

for some positive constant $\Lambda=\Lambda(\vec{p})$. We then prove (4.5) by using PalaisSmale properties of the sequence $\left(u_{\alpha}\right)_{\alpha}$. For any nonnegative, smooth function $\varphi$ with support in $B_{0}(2 R) \times \mathbb{R}^{n_{+}}$, we get

$$
\begin{aligned}
& \sum_{i=1}^{n}\left(\int_{\Omega_{\alpha}}\left|\frac{\partial u_{\alpha}}{\partial x_{i}}\right|^{p_{i}} \varphi d x+\int_{\Omega_{\alpha}}\left|\frac{\partial u_{\alpha}}{\partial x_{i}}\right|^{p_{i}-2} \frac{\partial u_{\alpha}}{\partial x_{i}} u_{\alpha} \frac{\partial \varphi}{\partial x_{i}} d x\right) \\
& \quad=\lambda_{\alpha} \int_{\Omega_{\alpha}}\left|u_{\alpha}\right|^{r_{\alpha}} \varphi d x+D J_{\alpha}\left(u_{\alpha}\right) \cdot\left(u_{\alpha} \varphi\right) \\
& \quad \leq \lambda_{\alpha}\left(\int_{\Omega_{\alpha}}\left|u_{\alpha}\right|^{p_{+}} \varphi d x+\int_{\Omega_{\alpha}} \varphi d x\right)+\mathrm{o}(1)
\end{aligned}
$$

as $\alpha \rightarrow+\infty$. For any $i=1, \ldots, n$, the functions $\left|\partial u_{\alpha} / \partial x_{i}\right|^{p_{i}-2} \partial u_{\alpha} / \partial x_{i}$ keep bounded in $L^{p_{i} /\left(p_{i}-1\right)}\left(\mathbb{R}^{n}\right)$, and thus converge, up to a subsequence, weakly to some function $\psi_{i}$ in $L^{p_{i} /\left(p_{i}-1\right)}\left(\mathbb{R}^{n}\right)$ as $\alpha \rightarrow+\infty$. Moreover, for any $i=$ $1, \ldots, n-n_{+}$, since $p_{i}<p_{+}$, by the compact embeddings in Rákosník [54], we get that the sequence $\left(u_{\alpha}\right)_{\alpha}$ converges to $u_{\infty}$ in $L^{p_{i}}(\operatorname{Supp} \varphi)$ and thus that

$$
\int_{\Omega_{\alpha}}\left|\frac{\partial u_{\alpha}}{\partial x_{i}}\right|^{p_{i}-2} \frac{\partial u_{\alpha}}{\partial x_{i}} u_{\alpha} \frac{\partial \varphi}{\partial x_{i}} d x \longrightarrow \int_{U} \psi_{i} u_{\infty} \frac{\partial \varphi}{\partial x_{i}} d x
$$

as $\alpha \rightarrow+\infty$. For any $\alpha$ and any $i=n-n_{+}+1, \ldots, n$, we get

$$
\left.\left|\int_{\Omega_{\alpha}}\right| \frac{\partial u_{\alpha}}{\partial x_{i}}\right|^{p_{+}-2} \frac{\partial u_{\alpha}}{\partial x_{i}} u_{\alpha} \frac{\partial \varphi}{\partial x_{i}} d x \mid \leq\left\|\frac{\partial u_{\alpha}}{\partial x_{i}}\right\|_{L^{p^{+}\left(\Omega_{\alpha}\right)}}^{p_{+}-1}\left\|u_{\alpha}\right\|_{L^{p_{+}\left(\Omega_{\alpha}\right)}}\left\|\frac{\partial \varphi}{\partial x_{i}}\right\|_{L^{\infty}\left(\mathbb{R}^{n}\right)} .
$$

By (4.6), (4.7), (4.11), and (4.12), passing to the limit into (4.10) as $\alpha \rightarrow+\infty$, we get

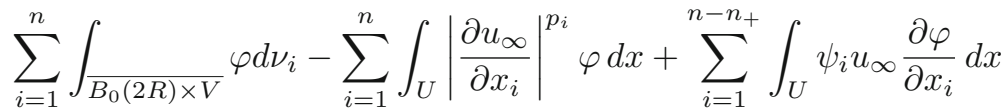

$$
\begin{aligned}
& \leq \lambda\left(\int_{U}\left|u_{\infty}\right|^{p_{+}} \varphi d x+\int_{\overline{B_{0}(2 R) \times V}} \varphi d \mu+\int_{\mathbb{R}^{n}} \varphi d x\right) \\
& +C \sum_{i=n-n_{+}+1}^{n}\left\|\frac{\partial \varphi}{\partial x_{i}}\right\|_{L^{\infty}\left(\mathbb{R}^{n}\right)}
\end{aligned}
$$

for some positive constant $C$ independent of $\varphi$. We claim that

$$
\sum_{i=1}^{n} \int_{U} \psi_{i} u_{\infty} \frac{\partial \varphi}{\partial x_{i}} d x+\sum_{i=1}^{n} \int_{U} \psi_{i} \frac{\partial u_{\infty}}{\partial x_{i}} \varphi d x=\lambda \int_{U}\left|u_{\infty}\right|^{p_{+}} \varphi d x .
$$

Since the function $u_{\infty}$ belongs to $D^{1, \vec{p}}(U)$ and since $\Omega_{\alpha} \rightarrow U$ as $\alpha \rightarrow+\infty$ in the sense of Definition 3.1, we get that there exist smooth functions $b_{\alpha}$ with compact support in $\Omega_{\alpha}$ converging to $u_{\infty}$ as $\alpha \rightarrow+\infty$ in $D^{1, \vec{p}}\left(\mathbb{R}^{n}\right)$, and thus also in $L^{p_{+}}\left(\mathbb{R}^{n}\right)$ by the continuity of the embedding of $D^{1, \vec{p}}\left(\mathbb{R}^{n}\right)$ into 
$L^{p_{+}}\left(\mathbb{R}^{n}\right)$. Since the sequence $\left(u_{\alpha}\right)_{\alpha}$ is Palais-Smale for the functionals $\left(J_{\alpha}\right)_{\alpha}$ defined in (4.1), it follows that

$$
\begin{aligned}
& \sum_{i=1}^{n} \int_{\Omega_{\alpha}}\left|\frac{\partial u_{\alpha}}{\partial x_{i}}\right|^{p_{i}-2} \frac{\partial u_{\alpha}}{\partial x_{i}} b_{\alpha} \frac{\partial \varphi}{\partial x_{i}} d x+\sum_{i=1}^{n} \int_{\Omega_{\alpha}}\left|\frac{\partial u_{\alpha}}{\partial x_{i}}\right|^{p_{i}-2} \frac{\partial u_{\alpha}}{\partial x_{i}} \frac{\partial b_{\alpha}}{\partial x_{i}} \varphi d x \\
& \quad=\lambda_{\alpha} \int_{\Omega_{\alpha}}\left|u_{\alpha}\right|^{r_{\alpha}-2} u_{\alpha} b_{\alpha} \varphi d x+D J_{\alpha}\left(u_{\alpha}\right) \cdot\left(b_{\alpha} \varphi\right) \\
& \quad=\lambda_{\alpha} \int_{\Omega_{\alpha}}\left|u_{\alpha}\right|^{r_{\alpha}-2} u_{\alpha} b_{\alpha} \varphi d x+\mathrm{o}(1)
\end{aligned}
$$

as $\alpha \rightarrow+\infty$. By (4.2), we get that the functions $\lambda_{\alpha}\left|u_{\alpha}\right|^{r_{\alpha}-2} u_{\alpha}$ keep bounded in $L^{p_{+} /\left(p_{+}-1\right)}\left(\mathbb{R}^{n}\right)$. Moreover the functions $\lambda_{\alpha}\left|u_{\alpha}\right|^{r_{\alpha}-2} u_{\alpha}$ converge, up to a subsequence, almost everywhere to $\lambda\left|u_{\infty}\right|^{p_{+}-2} u_{\infty}$ in $\mathbb{R}^{n}$ as $\alpha \rightarrow+\infty$. By standard integration theory, it follows that the functions $\lambda_{\alpha}\left|u_{\alpha}\right|^{r_{\alpha}-2} u_{\alpha}$ converge weakly to $\lambda\left|u_{\infty}\right|^{p_{+}-2} u_{\infty}$ in $L^{p_{+} /\left(p_{+}-1\right)}\left(\mathbb{R}^{n}\right)$. Passing to the limit into (4.15) as $\alpha \rightarrow+\infty$, we get (4.14). Increasing if necessary the constant $C$ in (4.13), it follows from (4.14) that

$$
\begin{aligned}
& \left.\sum_{i=1}^{n} \int_{\frac{B_{0}(2 R) \times V}{} \varphi d \nu_{i}-\sum_{i=1}^{n} \int_{U}\left|\frac{\partial u_{\infty}}{\partial x_{i}}\right|^{p_{i}} \varphi d x-\sum_{i=1}^{n} \int_{U} \psi_{i} \frac{\partial u_{\infty}}{\partial x_{i}} \varphi d x} \varphi d \mu+\int_{\mathbb{R}^{n}} \varphi d x\right)+C \sum_{i=n-n_{+}+1}^{n}\left\|\frac{\partial \varphi}{\partial x_{i}}\right\|_{L^{\infty}\left(\mathbb{R}^{n}\right)} .
\end{aligned}
$$

We let $\eta$ be a smooth cutoff function on $\mathbb{R}^{n-n_{+}}$such that $\eta=1$ in $B_{0}(1)$, $0 \leq \eta \leq 1$ in $B_{0}(2) \backslash B_{0}(1)$, and $\eta=0$ in $\mathbb{R}^{n-n_{+}} \backslash B_{0}(2)$. For any point $y=$ $\left(y_{1}, \ldots, y_{n-n_{+}}\right)$in $\overline{B_{0}(R)}$ and for any positive real number $\varepsilon$, we let $\varphi_{\varepsilon, y}$ be the function defined on $\mathbb{R}^{n}$ by

$$
\varphi_{\varepsilon, y}\left(x_{1}, \ldots, x_{n}\right)=\eta\left(\frac{1}{\varepsilon}\left(x_{1}-y_{1}\right), \ldots, \frac{1}{\varepsilon}\left(x_{n-n_{+}}-y_{n-n_{+}}\right)\right) .
$$

Plugging $\varphi=\varphi_{\varepsilon, \sigma, y}$ into (4.16), and passing to the limit as $\varepsilon \rightarrow 0$, we get

$$
\sum_{i=1}^{n} \nu_{i}\left(\bar{V}_{y}\right) \leq \lambda \mu\left(\bar{V}_{y}\right)
$$

where $\bar{V}_{y}$ is as in (4.3). By (4.9) and (4.17), we get that there holds either

$$
\mu\left(\bar{V}_{y}\right)=0 \quad \text { or } \quad \lambda \mu\left(\bar{V}_{y}\right)^{\frac{p_{+}}{n+p_{+}}} \geq \Lambda^{\frac{-n}{n+p_{+}}}
$$

for all points $y$ in $\mathbb{R}^{n-n_{+}}$. In particular, since the measure $\mu$ is finite, it follows from (4.18) that the index set $J$ in (4.8) is finite. Passing to the limit as $R \rightarrow+\infty$, by (4.6) and (4.8), we get the decomposition (4.4). Finally, (4.5) follows from (4.18).

\section{Proof of the main result}

In this section, we prove Theorem 1.1 by using Propositions 4.1 and 5.1 below. 
Proposition 5.1. Let $n \geq 3$ and $\vec{p}=\left(p_{1}, \ldots, p_{n}\right)$, and assume that $\sum_{i=1}^{n} 1 / p_{i}>$ 1 and $p_{+}=p^{*}$. Then there exists a positive constant $E_{0}=E_{0}(\vec{p})$ such that for any positive real number $\lambda$ and any $\vec{p}$-bubble $\left(B_{\alpha}\right)_{\alpha}$ of multiplier $\lambda$, there holds

$$
E\left(B_{\alpha}\right) \geq E_{0} \lambda^{-n / p_{+}},
$$

where $E\left(B_{\alpha}\right)$ is as in $(1.7)$.

Proof of Proposition 5.1. Without loss of generality, we may assume that there exists an index $n_{+}$such that $p_{n-n_{+}+1}=\cdots=p_{n}=p_{+}$and $p_{i}<p_{+}$for all $i \leq n-n_{+}$. We let $\lambda$ be a positive real number and $\left(B_{\alpha}\right)_{\alpha}$ be a $\vec{p}$-bubble of profile $u$ and multiplier $\lambda$. Since $p_{+}=p^{*}$, by the anisotropic Sobolev inequality in Troisi [65], we get

$$
\int_{\mathbb{R}^{n}}|u|^{p_{+}} d x \leq \Lambda \prod_{i=1}^{n}\left(\int_{\mathbb{R}^{n}}\left|\frac{\partial u}{\partial x_{i}}\right|^{p_{i}} d x\right)^{\frac{p_{+}}{n p_{i}}}
$$

for some positive constant $\Lambda$ independent of $u$. By Young's inequality, it follows that for any positive real number $\varepsilon$, there holds

$$
\begin{gathered}
\int_{\mathbb{R}^{n}}|u|^{p_{+}} d x \leq \frac{\Lambda}{n}\left(\left(n-n_{+}\right) \varepsilon^{\frac{-n_{+}}{n_{+}}} \prod_{i=1}^{n-n_{+}}\left(\int_{\mathbb{R}^{n}}\left|\frac{\partial u}{\partial x_{i}}\right|^{p_{i}} d x\right)^{\frac{p_{+}}{\left(n-n_{+}\right) p_{i}}}\right. \\
\left.+\varepsilon \sum_{i=n-n_{+}+1}^{n} \int_{\mathbb{R}^{n}}\left|\frac{\partial u}{\partial x_{i}}\right|^{p_{+}} d x\right) .
\end{gathered}
$$

On the other hand, since $u$ is a solution of problem (1.6), we get

$$
\sum_{i=1}^{n} \int_{\mathbb{R}^{n}}\left|\frac{\partial u}{\partial x_{i}}\right|^{p_{i}} d x=\lambda \int_{\mathbb{R}^{n}}|u|^{p_{+}} d x
$$

Taking $\varepsilon=\frac{n}{\Lambda \lambda}$, since $\sum_{i=1}^{n-n_{+}} \frac{1}{p_{i}}=\frac{n-n_{+}+p_{+}}{p_{+}}$, it follows from (5.2) and (5.3) that

$$
\sum_{i=1}^{n-n_{+}} \int_{\mathbb{R}^{n}}\left|\frac{\partial u}{\partial x_{i}}\right|^{p_{i}} d x \leq\left(\frac{\Lambda \lambda}{n}\right)^{\frac{n}{n_{+}}}\left(n-n_{+}\right)\left(\sum_{i=1}^{n-n_{+}} \int_{\mathbb{R}^{n}}\left|\frac{\partial u}{\partial x_{i}}\right|^{p_{i}} d x\right)^{\frac{n-n_{+}+p_{+}}{n-n_{+}}}
$$

We then easily get (5.1).

Proof of Theorem 1.1. Without loss of generality, we may assume that there exists an index $n_{+}$such that $p_{n-n_{+}+1}=\cdots=p_{n}=p_{+}$and $p_{i}<p_{+}$for all $i \leq n-n_{+}$. We let $\left(u_{\alpha}\right)_{\alpha}$ be a bounded Palais-Smale sequence for the functionals $\left(I_{\alpha}\right)_{\alpha}$ defined in (1.9). Passing if necessary to a subsequence, we may assume that $\left(u_{\alpha}\right)_{\alpha}$ converges weakly to a function $u_{\infty}$ in $D^{1, \vec{p}}(\Omega)$. Proceeding in the same way as in El Hamidi-Vétois [29], we can prove that $u_{\infty}$ is a solution of problem (1.2). By an easy adaptation of the argument in Brézis-Lieb [18] and by the growth condition (1.3), we also get that, up to a subsequence, $\left(u_{\alpha}^{0}\right)_{\alpha}$, where $u_{\alpha}^{0}=u_{\alpha}-u_{\infty}$, is Palais-Smale for the functionals $\left(J_{\alpha}\right)_{\alpha}$ defined in (4.1) with $\lambda_{\alpha}=\lambda$ and $\Omega_{\alpha}=\Omega$ for all $\alpha$, and that there holds 
$J_{\alpha}\left(u_{\alpha}^{0}\right)=I_{\alpha}\left(u_{\alpha}\right)-I_{\alpha}\left(u_{\infty}\right)+\mathrm{o}(1)$ as $\alpha \rightarrow+\infty$. If the sequence $\left(u_{\alpha}\right)_{\alpha}$ converges strongly, up to a subsequence, to the function $u_{\infty}$ in $D^{1, \vec{p}}(\Omega)$, then we get (1.11) and (1.12) with $k=0$. From now on, subtracting if necessary the weak limit $u_{\infty}$, we may assume that the sequence $\left(u_{\alpha}\right)_{\alpha}$ converges weakly but not strongly to 0 in $D^{1, \vec{p}}(\Omega)$, and that $\left(u_{\alpha}\right)_{\alpha}$ is Palais-Smale for the functionals $\left(J_{\alpha}\right)_{\alpha}$ with $\lambda_{\alpha}=\lambda$ and $\Omega_{\alpha}=\Omega$ for all $\alpha$. In particular, passing if necessary to a subsequence, we get

$$
\liminf _{\alpha \rightarrow+\infty} \sum_{i=1}^{n} \int_{\Omega}\left|\frac{\partial u_{\alpha}}{\partial x_{i}}\right|^{p_{i}} d x>0
$$

By Palais-Smale properties and by Hölder's inequality, it follows that

$$
\sum_{i=1}^{n} \int_{\Omega}\left|\frac{\partial u_{\alpha}}{\partial x_{i}}\right|^{p_{i}} d x=\int_{\Omega}\left|u_{\alpha}\right|^{r_{\alpha}} d x+\mathrm{o}(1) \leq|\Omega|^{\frac{p_{+}-r_{\alpha}}{p_{+}}}\left(\int_{\Omega}\left|u_{\alpha}\right|^{p_{+}} d x\right)^{\frac{r_{\alpha}}{p_{+}}}+\mathrm{o}(1)
$$

as $\alpha \rightarrow+\infty$, and thus that

$$
\liminf _{\alpha \rightarrow+\infty} \int_{\Omega}\left|u_{\alpha}\right|^{p_{+}} d x>0 .
$$

For any $\alpha$, we then define the concentration function $Q_{\alpha}: \mathbb{R}_{+} \rightarrow \mathbb{R}_{+}$by

$$
Q_{\alpha}(s)=\max _{y \in \bar{\Omega}} \int_{\mathcal{P}_{y}^{\vec{p}}(s)}\left|u_{\alpha}\right|^{p_{+}} d x
$$

where

$$
\mathcal{P}_{y}^{\vec{p}}(s)=\left\{\left(x_{1}, \ldots, x_{n}\right) \in \mathbb{R}^{n} ;\left|x_{i}-y_{i}\right|<s^{\frac{p_{+}-p_{i}}{p_{i}}} \quad \forall i \in\left\{1, \ldots, n-n_{+}\right\}\right\}
$$

for any positive real number $s$ and for any point $y=\left(y_{1}, \ldots, y_{n}\right)$ in $\mathbb{R}^{n}$. By (5.4) and since the domain $\Omega$ is bounded, we get the existence of two positive real numbers $s_{0}$ and $\delta_{0}$ such that there holds $Q_{\alpha}\left(s_{0}\right)>\delta_{0}$ for all $\alpha$. By the continuity of the functions $Q_{\alpha}$, it follows that there exists a sequence $\left(\mu_{\alpha}\right)_{\alpha}$ of real numbers in $\left(0, s_{0}\right)$ such that there holds $Q_{\alpha}\left(\mu_{\alpha}\right)=\delta_{0}$ for all $\alpha$. We let $x_{\alpha}$ be a point in $\bar{\Omega}$ for which $Q_{\alpha}\left(\mu_{\alpha}\right)$ is reached, so that

$$
\max _{y \in \bar{\Omega}} \int_{\mathcal{P}_{y}^{\vec{p}}\left(\mu_{\alpha}\right)}\left|u_{\alpha}\right|^{p_{+}} d x=\int_{\mathcal{P}_{x_{\alpha}}^{\vec{p}}\left(\mu_{\alpha}\right)}\left|u_{\alpha}\right|^{p_{+}} d x=\delta_{0}
$$

for all $\alpha$. We claim that if the constant $\delta_{0}$ is chosen small enough, then the sequence $\left(\mu_{\alpha}\right)_{\alpha}$ converges to 0 . Indeed, if not the case, then for any $\varepsilon>0$, there exists $s_{\varepsilon}>0$ such that for any $\alpha$ and any point $y$ in $\bar{\Omega}$, there holds

$$
\int_{\mathcal{P}_{y}^{\vec{p}}\left(s_{\varepsilon}\right)}\left|u_{\alpha}\right|^{p_{+}} d x \leq \varepsilon .
$$

By Proposition 4.1 with $\lambda_{\alpha}=\lambda$ and $\Omega_{\alpha}=\Omega$ for all $\alpha$ (in this case, we can easily drop the segment condition), it follows from (5.6) that the sequence $\left(u_{\alpha}\right)_{\alpha}$ converges in fact strongly to 0 in $L^{p_{+}}(\Omega)$. There is a contradiction with (5.4), and this proves our claim, namely that if the constant $\delta_{0}$ is chosen small 
enough, then the sequence $\left(\mu_{\alpha}\right)_{\alpha}$ converges to 0 . For any $\alpha$, we then define the function $\widetilde{u}_{\alpha}$ by

$$
\widetilde{u}_{\alpha}=\mu_{\alpha} u_{\alpha} \circ\left(\tau_{\mu_{\alpha}, x_{\alpha}}^{\vec{p}}\right)^{-1}
$$

where $\tau_{\mu_{\alpha}, x_{\alpha}}^{\vec{p}}$ is as in (1.5). As well as $\left(u_{\alpha}\right)_{\alpha}$, the sequence $\left(\widetilde{u}_{\alpha}\right)_{\alpha}$ is Palais-Smale for the functionals $\left(J_{\alpha}\right)_{\alpha}$ with $\lambda_{\alpha}=\lambda \mu_{\alpha}^{p_{+}-r_{\alpha}}$ and $\Omega_{\alpha}=\tau_{\mu_{\alpha}, x_{\alpha}}^{\vec{p}}(\Omega)$ for all $\alpha$. Passing if necessary to a subsequence, we may assume that the sequence $\left(\lambda_{\alpha}\right)_{\alpha}$ converges to a nonnegative real number $\widetilde{\lambda}$. By an easy change of variable, we also find

$$
\lambda_{\alpha} \int_{\Omega_{\alpha}}\left|\widetilde{u}_{\alpha}\right|^{r_{\alpha}} d x=\lambda \int_{\Omega}\left|u_{\alpha}\right|^{r_{\alpha}} d x
$$

and

$$
\lambda_{\alpha}^{\frac{p_{+}}{p_{+}-1}} \int_{\Omega_{\alpha}}\left|\widetilde{u}_{\alpha}\right|^{\frac{\left(r_{\alpha}-1\right) p_{+}}{p_{+}-1}} d x=\lambda^{\frac{p_{+}}{p_{+}-1}} \int_{\Omega}\left|u_{\alpha}\right|^{\frac{\left(r_{\alpha}-1\right) p_{+}}{p_{+}-1}} d x .
$$

It follows from (5.7) and (5.8) that $\left(\widetilde{u}_{\alpha}\right)_{\alpha}$ is a bounded Palais-Smale sequence for the functionals $\left(J_{\alpha}\right)_{\alpha}$. Passing if necessary to a subsequence, since the domain $\Omega$ is asymptotically $\vec{p}$-stable, we may assume that there exists an open subset $U$, either empty or satisfying the segment property, such that $\Omega_{\alpha} \rightarrow U$ as $\alpha \rightarrow+\infty$ in the sense of Definition 3.1. Moreover, since $\Omega$ is bounded and since the maps $\tau_{\mu_{\alpha}, x_{\alpha}}^{\vec{p}}$ do not rescale the domain in critical directions, we get that the sets $\Omega_{\alpha}$ and $U$ are included in $\mathbb{R}^{n-n_{+}} \times V$ for some bounded, open subset $V$ independent of $\alpha$. By Proposition 4.1, we get the existence of a function $\widetilde{u}$ in $D^{1, \vec{p}}(U)(\widetilde{u}=0$ in case $U=\emptyset)$ and a finite number $k$ of distinct points $y_{j}=\left(y_{1}^{j}, \ldots, y_{n-n_{+}}^{j}\right)$ in $\mathbb{R}^{n-n_{+}}, j=1, \ldots, k$, and nonnegative finite measures $\xi_{j}$ with support in $\bar{V}_{y_{j}}, j=1, \ldots, k$, such that, up to a subsequence, there holds

$$
\left|\widetilde{u}_{\alpha}\right|^{p_{+}} \longrightarrow|\widetilde{u}|^{p_{+}}+\sum_{j=1}^{k} \xi_{j}
$$

as $\alpha \rightarrow+\infty$ in the sense of measures on compact subsets of $\mathbb{R}^{n}$. If $\widetilde{\lambda}=0$, then $k=0$. Otherwise, in case $\widetilde{\lambda}>0$, we claim that if the constant $\delta_{0}$ is chosen small enough, then $k=0$. We assume by contradiction that $k \neq 0$. By $(5.5)$ and by an easy change of variable, for any $\alpha$ and $j=1, \ldots, k$, we get

$$
\int_{\mathcal{P} \vec{p}_{j}(1)}\left|\widetilde{u}_{\alpha}\right|^{p_{+}} d x \leq \delta_{0}
$$

Since there holds $\bar{V}_{y_{j}} \subset \mathcal{P}_{y_{j}}^{\vec{p}}(1)$, passing to the limit into (5.9) as $\alpha \rightarrow+\infty$, it follows that

$$
\int_{\mathcal{P}_{y_{j}}^{\vec{p}}(1)}|\widetilde{u}|^{p_{+}} d x+\xi_{j}\left(\bar{V}_{y_{j}}\right) \leq \delta_{0} .
$$

On the other hand, by (4.5), we get $\xi_{j}\left(\bar{V}_{y_{j}}\right) \geq \Lambda_{0} \widetilde{\lambda}^{-\left(n+p_{+}\right) / p_{+}}$. Since there

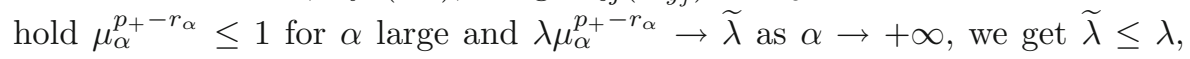


and thus $\xi_{j}\left(\bar{V}_{y_{j}}\right) \geq \Lambda_{0} \lambda^{-\left(n+p_{+}\right) / p_{+}}$. There is a contradiction with (5.10) in case $\delta_{0}<\Lambda_{0} \lambda^{-\left(n+p_{+}\right) / p_{+}}$. This ends the proof of our claim, namely that if the constant $\delta_{0}$ is chosen small enough, then $k=0$. By Radon's theorem, it follows that the sequence $\left(\widetilde{u}_{\alpha}\right)_{\alpha}$ converges to $\widetilde{u}$ in $L_{\text {loc }}^{p_{+}}\left(\mathbb{R}^{n}\right)$. By $(5.5)$ and by an easy change of variable, for any $\alpha$, we get

$$
\int_{\mathcal{P}_{0}^{\vec{p}}(1)}\left|\widetilde{u}_{\alpha}\right|^{p_{+}} d x=\delta_{0} .
$$

Passing to the limit into (5.11) as $\alpha \rightarrow+\infty$, it follows that

$$
\int_{\mathcal{P}_{0}^{\vec{p}}(1)}|\widetilde{u}|^{p_{+}} d x=\delta_{0},
$$

and thus that the function $\widetilde{u}$ is not identically zero. In particular, the domain $U$ is not empty. The end of the proof follows Struwe [62] (see El HamidiVétois [29] for the case of the anisotropic Laplace operator with $p_{+}<p^{*}$ ), and we sketch it for sake of completeness. We prove that the function $\widetilde{u}$ is a solution of problem (1.6) with $\lambda=\widetilde{\lambda}$, so that we can define the $\vec{p}$-bubble $\left(B_{\alpha}^{1}\right)_{\alpha}, B_{\alpha}^{1}=\mu_{\alpha}^{-1} \widetilde{u} \circ \tau_{\mu_{\alpha}, x_{\alpha}}^{\vec{p}}$. We then prove that there exists a sequence $\left(R_{\alpha}^{1}\right)_{\alpha}$ converging strongly to 0 in $D^{1, \vec{p}}\left(\mathbb{R}^{n}\right)$ such that, up to a subsequence, $\left(u_{\alpha}^{1}\right)_{\alpha}$, where $u_{\alpha}^{1}=u_{\alpha}-B_{\alpha}^{1}+R_{\alpha}^{1}$, is Palais-Smale for the functionals $\left(J_{\alpha}\right)_{\alpha}$ and satisfies $J_{\alpha}\left(u_{\alpha}^{1}\right)=J_{\alpha}\left(u_{\alpha}\right)-E\left(B_{\alpha}^{1}\right)+\mathrm{o}(1)$ as $\alpha \rightarrow+\infty$. We iterate the above arguments in order to construct the $\vec{p}$-bubbles $\left(B_{\alpha}^{1}\right)_{\alpha}, \ldots,\left(B_{\alpha}^{k}\right)_{\alpha}$. Our induction has to stop for some finite number $k$ since, by Proposition 5.1, the energy of $\vec{p}$-bubbles is bounded from below by a positive constant which only depends on $\lambda$ and on the anisotropic configuration $\vec{p}$. We finally obtain (1.11) and (1.12). Moreover, in case the functions $u_{\alpha}$ are nonnegative, their weak limit $u_{\infty}$ is nonnegative, and by considering the functions $\max \left(u_{\alpha}^{j}-B_{\alpha}^{j}, 0\right)$, we prove that the $\vec{p}$-bubbles $\left(B_{\alpha}^{1}\right)_{\alpha}, \ldots,\left(B_{\alpha}^{k}\right)_{\alpha}$ are also nonnegative (see [29]). This ends the proof of Theorem 1.1 .

\section{Acknowledgments}

The author is very grateful to Emmanuel Hebey for many helpful remarks and suggestions during the preparation of the manuscript.

\section{References}

[1] Adams, R.A., Fournier, J.J.F.: Sobolev spaces. In: Pure and Applied Mathematics, 2nd edn., vol. 140. Academic Press, New York (2003)

[2] Alves, C.O.: Positive solutions to quasilinear equations involving critical exponent on perturbed annular domains. Electron. J. Differ. Equ. 13, 13 pp (2005, electronic)

[3] Alves, C.O., Ding, Y.H.: Multiplicity of positive solutions to a $p$-Laplacian equation involving critical nonlinearity. J. Math. Anal. Appl. 279(2), 508-521 (2003) 
[4] Alves, C.O., El Hamidi, A.: Existence of solution for a anisotropic equation with critical exponent. Differ. Integral Equ. 21(1), 25-40 (2008)

[5] Antontsev, S., Díaz, J.I., Shmarev, S.: Energy methods for free boundary problems: applications to nonlinear PDEs and fluid mechanics. In: Progress in Nonlinear Differential Equations and their Applications, vol. 48. Birkhäuser, Boston (2002)

[6] Antontsev, S., Shmarev, S.: Elliptic equations and systems with nonstandard growth conditions: existence, uniqueness and localization properties of solutions. Nonlinear Anal. 65(4), 728-761 (2006)

[7] Antontsev, S., Shmarev, S.: Elliptic equations with anisotropic nonlinearity and nonstandard growth conditions. In: Handbook of Differential Equations: Stationary Partial Differential Equations, vol. 3. Elsevier, Amsterdam (2006)

[8] Arioli, G., Gazzola, F.: Some results on $p$-Laplace equations with a critical growth term. Differ. Integral Equ. 11(2), 311-326 (1998)

[9] Bahri, A., Coron, J.-M.: On a nonlinear elliptic equation involving the critical Sobolev exponent: the effect of the topology of the domain. Commun. Pure Appl. Math. 41(3), 253-294 (1988)

[10] Bear, J.: Dynamics of Fluids in Porous Media. American Elsevier, New York (1972)

[11] Bendahmane, M., Karlsen, K.H.: Renormalized solutions of an anisotropic reaction-diffusion-advection system with $L^{1}$ data. Commun. Pure Appl. Anal. 5(4), 733-762 (2006)

[12] Bendahmane, M., Karlsen, K.H.: Nonlinear anisotropic elliptic and parabolic equations in $\mathbb{R}^{N}$ with advection and lower order terms and locally integrable data. Potential Anal. 22(3), 207-227 (2005)

[13] Bendahmane, M., Langlais, M., Saad, M.: On some anisotropic reaction-diffusion systems with $L^{1}$-data modeling the propagation of an epidemic disease. Nonlinear Anal. 54(4), 617-636 (2003)

[14] Besov, O.V.: Embeddings of an anisotropic Sobolev space for a domain with a flexible horn condition. Trudy Mat. Inst. Steklov. vol. 181, pp. 3-14 (1988) (Russian); English transl., Proc. Steklov Inst. Math., no. 4, 1-13 (1989)

[15] Boccardo, L., Gallouët, T., Marcellini, P.: Anisotropic equations in $L^{1}$. Differ. Integral Equ. 9(1), 209-212 (1996)

[16] Boccardo, L., Marcellini, P., Sbordone, C.: $L^{\infty}$-regularity for variational problems with sharp nonstandard growth conditions. Boll. Un. Mat. Ital. A (7) 4(2), 219-225 (1990)

[17] Brézis, H., Coron, J.-M.: Convergence of solutions of H-systems or how to blow bubbles. Arch. Ration. Mech. Anal. 89(1), 21-56 (1985)

[18] Brézis, H., Lieb, E.: A relation between pointwise convergence of functions and convergence of functionals. Proc. Am. Math. Soc. 88(3), 486-490 (1983) 
[19] Brézis, H., Nirenberg, L.: Positive solutions of nonlinear elliptic equations involving critical Sobolev exponents. Commun. Pure Appl. Math. 36(4), 437$477(1983)$

[20] Cianchi, A.: Symmetrization in anisotropic elliptic problems. Commun. Partial Differ. Equ. 32(4-6), 693-717 (2007)

[21] D'Ambrosio, L.: Liouville theorems for anisotropic quasilinear inequalities. Nonlinear Anal. 70(8), 2855-2869 (2009)

[22] Demengel, F., Hebey, E.: On some nonlinear equations involving the $p$-Laplacian with critical Sobolev growth. Adv. Differ. Equ. 3(4), 533-574 (1998)

[23] Demengel, F., Hebey, E.: On some nonlinear equations involving the $p$-Laplacian with critical Sobolev growth and perturbation terms. Appl. Anal. 72(1-2), 75-109 (1999)

[24] Di Castro, A.: Existence and regularity results for anisotropic elliptic problems. Adv. Nonlinear Stud. 9, 367-393 (2009)

[25] Di Castro, A., Montefusco, E.: Nonlinear eigenvalues for anisotropic quasilinear degenerate elliptic equations. Nonlinear Anal. 70(11), 4093-4105 (2009)

[26] Druet, O., Hebey, E., Robert, F.: Blow-up theory for elliptic PDEs in Riemannian geometry. In: Mathematical Notes, vol. 45. Princeton University Press, Princeton (2004)

[27] El Hamidi, A., Rakotoson, J.-M.: On a perturbed anisotropic equation with a critical exponent. Ricerche Mat. 55(1), 55-69 (2006)

[28] El Hamidi, A., Rakotoson, J.-M.: Extremal functions for the anisotropic Sobolev inequalities. Ann. Inst. H. Poincaré Anal. Non Linéaire 24(5), 741-756 (2007)

[29] El Hamidi, A., Vétois, J.: Sharp Sobolev asymptotics for critical anisotropic equations. Arch. Ration. Mech. Anal. 192(1), 1-36 (2009)

[30] Filippucci, R., Pucci, P., Robert, F.: On a $p$-Laplace equation with multiple critical nonlinearities. J. Math. Pures Appl. (9) 91(2), 156-177 (2009)

[31] Fragalà, I., Gazzola, F., Kawohl, B.: Existence and nonexistence results for anisotropic quasilinear elliptic equations. Ann. Inst. H. Poincaré Anal. Non Linéaire 21(5), 715-734 (2004)

[32] Fragalà, I., Gazzola, F., Lieberman, G.: Regularity and nonexistence results for anisotropic quasilinear elliptic equations in convex domains. Discrete Contin. Dyn. Syst. 280-286 (2005)

[33] Franchi, B., Lanconelli, E., Serrin, J.: Existence and uniqueness of nonnegative solutions of quasilinear equations in $\mathbb{R}^{n}$. Adv. Math. 118(2), 177-243 (1996)

[34] García-Melián, J., Rossi, J.D., Sabina de Lis, J.C.: Large solutions to an anisotropic quasilinear elliptic problem. Ann. Mat. Pura Appl. (4) 189(4), 689$712(2010)$ 
[35] Gazzola, F.: Critical growth quasilinear elliptic problems with shifting subcritical perturbation. Differ. Integral Equ. 14(5), 513-528 (2001)

[36] Ghoussoub, N.: Duality and perturbation methods in critical point theory. In: Cambridge Tracts in Mathematics, vol. 107, Cambridge University Press, Cambridge (1993) (With appendices by David Robinson)

[37] Guedda, M., Véron, L.: Quasilinear elliptic equations involving critical Sobolev exponents. Nonlinear Anal. 13(8), 879-902 (1989)

[38] Haškovec, J., Schmeiser, C.: A note on the anisotropic generalizations of the Sobolev and Morrey embedding theorems. Monatsh. Math. 158(1), 71-79 (2009)

[39] Kolod $\breve{\imath}$, I.M.: An estimate for the maximum modulus of generalized solutions of the Dirichlet problem for elliptic equations in divergence form. Ukrain. Mat. Zh. 47(5), 635-648 (1995) (Ukrainian); English transl. in Ukrainian Math. J. 47(5) (1995)

[40] Kruzhkov, S.N., Kolodı̈ı, I.M.: On the theory of anisotropic Sobolev spaces. Uspekhi Mat. Nauk 38(2), 207-208 (1983) (Russian); English transl., Russ. Math. Surveys 38(2), 188-189 (1983)

[41] Kruzhkov, S.N., Korolev, A.G.: On embedding theory for anisotropic function spaces. Dokl. Akad. Nauk SSSR 285(5), 1054-1057 (1985) (Russian); English transl., Soviet Math. Dokl. 32(3), 829-832 (1985)

[42] Li, F.Q.: Anisotropic elliptic equations in $L^{m}$. J. Convex Anal. 8(2), 417$422(2001)$

[43] Lieberman, G.M.: Gradient estimates for a new class of degenerate elliptic and parabolic equations. Ann. Scuola Norm. Sup. Pisa Cl. Sci. (4) 21(4), 497$522(1994)$

[44] Lieberman, G.M.: Gradient estimates for anisotropic elliptic equations. Adv. Differ. Equ. 10(7), 767-812 (2005)

[45] Lions, P.-L.: The concentration-compactness principle in the calculus of variations. The limit case. I. Rev. Mat. Iberoamericana 1(1), 145-201 (1985)

[46] Lions, P.-L.: The concentration-compactness principle in the calculus of variations. The limit case. II. Rev. Mat. Iberoamericana 1(2), 45-121 (1985)

[47] Lu, V.-T.: On imbedding theorems for spaces of functions with partial derivatives of various degrees of summability. Vestnik Leningrad. Univ. 16(7), 23-37 (Russian) (1961)

[48] Marcellini, P.: Regularity and existence of solutions of elliptic equations with p, q-growth conditions. J. Differ. Equ. 90(1), 1-30 (1991)

[49] Maz'ya, V.G., Poborchi, S.V.: Differentiable Functions on Bad Domains. World Scientific Publishing Co. Inc., River Edge (1997) 
[50] Mihăilescu, M., Pucci, P., Rădulescu, V.: Eigenvalue problems for anisotropic quasilinear elliptic equations with variable exponent. J. Math. Anal. Appl. 340(1), 687-698 (2008)

[51] Mihăilescu, M., Rădulescu, V., Tersian, S.: Eigenvalue problems for anisotropic discrete boundary value problem. J. Difference Equ. Appl. 15(6), 557-567 (2009)

[52] Namlyeyeva, Y.V., Shishkov, A.E., Skrypnik, I.I.: Isolated singularities of solutions of quasilinear anisotropic elliptic equations. Adv. Nonlinear Stud. 6(4), 617-641 (2006)

[53] Nikol'skiı̌, S.M.: Imbedding, continuation and approximation theorems for differentiable functions of several variables, Uspehi Mat. Nauk 16(5), 63-114 (Russian) (1961)

[54] Rákosník, J.: Some remarks to anisotropic Sobolev spaces. I. Beiträge Anal. (13), 55-68 (1979)

[55] Rákosník, J.: Some remarks to anisotropic Sobolev spaces. II. Beiträge Anal. (15), 127-140 (1980)

[56] Rey, O.: A multiplicity result for a variational problem with lack of compactness. Nonlinear Anal. 13(10), 1241-1249 (1989)

[57] Rey, O.: The role of the Green's function in a nonlinear elliptic equation involving the critical Sobolev exponent. J. Funct. Anal. 89(1), 1-52 (1990)

[58] Sacks, J., Uhlenbeck, K.K.: The existence of minimal immersions of 2-spheres. Ann. Math. (2) 113(1), 1-24 (1981)

[59] Saintier, N.: Asymptotic estimates and blow-up theory for critical equations involving the $p$-Laplacian. Calc. Var. Partial Differ. Equ. 25(3), 299-331 (2006)

[60] Schoen, R.M.: Variational theory for the total scalar curvature functional for Riemannian metrics and related topics. In: Topics in calculus of variations (Montecatini Terme, 1987). Lecture Notes in Math., vol. 1365, pp. 120-154. Springer, Berlin (1989)

[61] Skrypnik, I.I.: Removability of an isolated singularity for anisotropic elliptic equations with absorption. Math. Sb. 199(7), 1033-1050 (2008)

[62] Struwe, M.: A global compactness result for elliptic boundary value problems involving limiting nonlinearities. Math. Z. 187(4), 511-517 (1984)

[63] Struwe, M.: Variational Methods: Applications to Nonlinear Partial Differential Equations and Hamiltonian Systems. Springer, Berlin (1990)

[64] Tersenov, A.S., Tersenov, A.S.: The problem of Dirichlet for anisotropic quasilinear degenerate elliptic equations. J. Differ. Equ. 235(2), 376-396 (2007)

[65] Troisi, M.: Teoremi di inclusione per spazi di Sobolev non isotropi. Ricerche Mat. 18, 3-24 (1969, Italian)

[66] Vétois, J.: A priori estimates for solutions of anisotropic elliptic equations. Nonlinear Anal. 71(9), 3881-3905 (2009) 
[67] Vétois, J.: Asymptotic stability, convexity, and Lipschitz regularity of domains in the anisotropic regime. Commun. Contemp. Math. 12(1), 35-53 (2010)

[68] Vétois, J.: Existence and regularity for critical anisotropic equations with critical directions. Adv. Differ. Equ. 16(1-2) (2010, to appear)

[69] Vétois, J.: Strong maximum principles for anisotropic elliptic and parabolic equations (2010, preprint)

[70] Wente, H.C.: Large solutions to the volume constrained Plateau problem. Arch. Ration. Mech. Anal. 75(1), 59-77 (1980/1981)

[71] Yan, S.: A global compactness result for quasilinear elliptic equation involving critical Sobolev exponent. Chin. J. Contemp. Math. 16(3), 227-234 (1995)

Jérôme Vétois

Laboratoire J.-A. Dieudonné, CNRS UMR 6621,

Université de Nice Sophia Antipolis,

Parc Valrose, 06108 Nice Cedex 2, France

e-mail: vetois@unice.fr

Received: 25 February 2010.

Revised: 18 September 2010.

Accepted: 12 October 2010. 